ARTICLE

\title{
Intratumoral generation of photothermal gold nanoparticles through a vectorized biomineralization of ionic gold
}

\author{
Aaron S. Schwartz-Duval (10) 1,2,3, Christian J. Konopka (1) 1,2, Parikshit Moitra ${ }^{4,5,6}$, Enrique A. Daza ${ }^{1,2,3}$, \\ Indrajit Srivastava ${ }^{1,2,3}$, Elyse V. Johnson7, Taylor L. Kampert ${ }^{1,2,3}$, Stanley Fayn ${ }^{1,2}$, Anand Haran ${ }^{1,2}$, \\ Lawrence W. Dobrucki (i) ${ }^{1,2}$ \& Dipanjan Pan (i) 1,2,3,4,5,6凶
}

Various cancer cells have been demonstrated to have the capacity to form plasmonic gold nanoparticles when chloroauric acid is introduced to their cellular microenvironment. But their biomedical applications are limited, particularly considering the millimolar concentrations and longer incubation period of ionic gold. Here, we describe a simplistic method of intracellular biomineralization to produce plasmonic gold nanoparticles at micromolar concentrations within $30 \mathrm{~min}$ of application utilizing polyethylene glycol as delivery vector for ionic gold. We have characterized this process for intracellular gold nanoparticle formation, which progressively accumulates proteins as the ionic gold clusters migrate to the nucleus. This nano-vectorized application of ionic gold emphasizes its potential biomedical opportunities while reducing the quantity of ionic gold and required incubation time. To demonstrate its biomedical potential, we further induce in-situ biosynthesis of gold nanoparticles within MCF7 tumor mouse xenografts which is followed by its photothermal remediation.

\footnotetext{
${ }^{1}$ Department of Bioengineering, University of Illinois, Urbana-Champaign, Urbana, IL 61801, USA. ${ }^{2}$ Beckman Institute, University of Illinois, UrbanaChampaign, Urbana, IL 61801, USA. ${ }^{3}$ Biomedical Research Center, Carle Foundation Hospital, Urbana, IL, USA. ${ }^{4}$ Department of Diagnostic Radiology and Nuclear Medicine, University of Maryland Baltimore School of Medicine, Baltimore, MD 21201, USA. ${ }^{5}$ Department of Pediatrics, Center for Blood Oxygen Transport and Hemostasis, University of Maryland Baltimore School of Medicine, Health Sciences Research Facility III, 670 W Baltimore St., Baltimore, MD 21201, USA. ${ }^{6}$ Department of Chemical, Biochemical and Environmental Engineering, University of Maryland Baltimore County, Interdisciplinary Health Sciences Facility, 1000 Hilltop Circle Baltimore, Baltimore, MD 21250, USA. ${ }^{7}$ Cytoviva inc., Auburn 36830, USA. ${ }^{凶}$ email: dipanjan@som.umaryland.edu
} 
G old nanoparticles have been extensively utilized for biological applications due to their simplistic and tunable chemistry which provides the ability to control and modify size, morphology, and surface functionality; combined with their morphology-dependent ${ }^{1-4}$. Typically, generating gold nanoparticles require, at the minimum, chloroauric acid as a precursor material and a reducing agent for catalyzing the reductive nucleation. Variations in the reducing agent molecule as well as reaction conditions (e.g. $\mathrm{pH}$, temperature, incubation time) will result in different morphologies, sizes, and functionalities of the resulting nanoparticles ${ }^{5-8}$. For biomedical applications, these variations in morphology, size, and functionality are directly correlated to their cellular internalization ${ }^{9-11}$, biodistribution, biological half-life ${ }^{11-13}$, renal secretion ${ }^{11-15}$, and plasmon optical properties ${ }^{1-8}$. Because of their plasmon-optical properties, gold nanoparticles are inherently theranostic, providing imaging contrast through near infrared (NIR) $16,17,15,16$, fluorescence ${ }^{18,19}, \mathrm{X}$-Ray ${ }^{20,21}$, photoacoustic ${ }^{22-24}$, and Raman enhancement ${ }^{25,26}$. This plasmonic optical property therefore allows gold nanoparticles to act as a vehicle to enhance ablative therapies both for phototherma $2^{27,28}$, and X-ray radiation ${ }^{29-31}$.

On the other hand, the bio-inertness of gold nanoparticles combined with the diverse range of applicable reductant molecules has allowed researchers to develop biomimetic gold nanoparticles using biomolecules as scaffolds for its reduction ${ }^{32-35}$. Therefore, this approach requires no additional steps for bioconjugation since the reducing biomolecules remain attached as capping agents to the formed nanoparticles. Further to this, if cellular machinery are used to generate gold nanoparticles, the resulting plasmon-optical properties could act as a reporter of the affecting cell phenotype. This capability to generate gold nanoparticles via cellular biomolecules has already been demonstrated previously in mammalian cells $s^{36-47}$. The precise mechanism for this cellular bioreduction of gold nanoparticles is not fully understood, however, these treatments have been known to negatively impact cell health through cellular responses via shock and stress-related proteins ${ }^{42}$, decrease in viability ${ }^{43,44}$, and even cell fixation ${ }^{44}$. These previous works, wherein ionic gold was applied diffusely to generate gold nanoparticles via cellular mechanisms, could not be directly applied for cellular diagnostics or therapeutics, as the process is so deleterious to cell health and not cell-specific. However, this process of cellular bioreduction forming gold nanoparticles has been marketed as a means for generating biomimetic and biofunctional gold nanoparticles, which could potentially be applied to different physiological systems. One such recent work by A.V. Singh et al. demonstrated the photothermal effects from intracellular gold, reduced in vitro through the addition of prefabricated gold nanoparticle seeds ${ }^{36}$. This work demonstrated that the process of biomineralization could easily be modified through suitable tuning in the recipe of precursor materials.

In this work, we hypothesize that if ionic gold are deployed to cells in discrete nanoscaled packets, this can largely reduce the concentration of gold necessary for the reduction, as well as decrease the time frame and overall impact on cell health, thereby broadening its use for diagnostic and therapeutic purposes. Accordingly, a simplistic delivery vehicle has been demonstrated herein for deploying ionic gold, which indicate intracellular nanoparticle formation within a time span of $\sim 30 \mathrm{~min}$, much less than the previous literature reports, with concentrations lower than previous applications, in complete cell media. Additionally, we have identified proteins and their respective subcellular locations involved in the cellular bioreduction of gold nanoparticles providing larger insight into this potential reduction pathway. The process of sequential and progressive reduction of gold nanoparticle has been shown to occur initially via secreted proteins in the extracellular space and finally within the nucleus for terminal reduction. Because this approach has minimal impact on protein expression/integrity and some of the bound proteins are nucleotide binding, there is a strong potential of the developed gold nanoparticles to be used for theranostic application to provide photothermal ablation directly to the nucleotides. Thus, we demonstrate a theranostic application through a MCF-7 cancer xenograft model, wherein fluorescent and photothermally active gold nanoparticles are formed intracellularly to remediate cancer xenografts.

\section{Results}

Vehicle design and characterization for ionic gold delivery. For intracellular gold nanoparticle formation, all of the constituents (sans chloroauric acid) are innately present in cellular systems. This intracellular gold nanoparticle formation has been demonstrated previously in mammalian cells, however, only through bulk diffusive treatments, lasting more than $24 \mathrm{~h}$, and typically those are in solutions which contain no cell nutrients (such as DPBS) ${ }^{35-47}$. These treatment scenarios are non-ideal for minimizing the impact on cellular health. To minimize the caustic properties of chloroauric acid, we aim to deploy ionic gold salts in discrete nano-scaled clusters. It was hypothesized that if we are successful in deploying ionic gold in discrete nanoscaled clusters, it could potentially allow us to reduce the overall dosage, required for intracellular gold nanoparticle (GNP) formation, and in turn, reduce the impact on cellular physiology. In order to successfully enable intracellular reduction of ionic gold by the action of cellular biomolecules, it is necessary that the ionic gold delivery vehicle: (a) is made of material that will not spontaneously reduce ionic gold, (b) will contain the ionic gold in discrete nanoscale packets, (c) will have the capacity to interact with the cells, and (d) that cellular biomolecules will have access to the gold ions for nanoparticle formation. While chloroauric acid can be readily reduced by a large varieties of functional groups, it is not readily reduced by ether groups, carbon-carbon single bonds, nor under acidic $\mathrm{pH}$ conditions. With this knowledge in hand, hydroxyl terminated polyethylene glycol (PEG) was used as the ionic $\mathrm{Au}^{3+}$ delivery vehicle. The rationale behind this was that PEG is known to cluster with ionic gold in acidic $\mathrm{pH}$ solutions ${ }^{6}$, and will not cause spontaneous gold nanoparticle (GNP) formation. We do not suspect that PEG would induce spontaneous GNP formation as polyol-based $\mathrm{Au} / \mathrm{Ag}$ nanoparticle synthesis, where PEG could function as a reductant, typically require much higher temperatures $\left(160^{\circ} \mathrm{C}\right)$ than utilized for cell culture $\left(37^{\circ} \mathrm{C}\right)^{48}$. In a typical process for the formation of ionic gold delivery vehicle, $\mathrm{HAuCl}_{4}$ $(14 \mu \mathrm{mol})$ and PEG $(5 \mu \mathrm{mol})$ were co-clustered in a sealed glass vial with $2 \mathrm{ml}$ of carbon filtered deionized water $(\mathrm{pH}=4)$. We observed that PEG molecules formed clusters in acidic conditions when compared to neutral conditions. In addition, positively charged gold ions were seen to preferentially localize within these clusters (Fig. 1a, b). This mixture of ionic gold and PEG was then incubated in a water bath kept at $37^{\circ} \mathrm{C}$ for $30 \mathrm{~min}$ to ensure thermal equilibrium achieved before dilution for analysis and/or cell treatment. A UV-Vis electromagnetic spectra confirmed that the gold in these Au-PEG clusters was ionic and not reduced in nature (Fig. 1c) due to the lack of absorbance peaks that are characteristic of plasmon formation. This spectrum revealed an absorbance spectra typical of ionic gold (Supplementary Fig. 1a), with no observable peak in the $500-700 \mathrm{~nm}$ range that is characteristic of the surface plasmonic resonance of reduced gold ${ }^{1-4}$. Additionally, no visible color change or precipitant formation was observed if the solution was left undisturbed on the benchtop (RT) for 30 days (all treatments and samples used for analysis were made fresh). The discrete Au-PEG clusters were $288 \pm 46 \mathrm{~nm}$ in diameter, as quantified by 


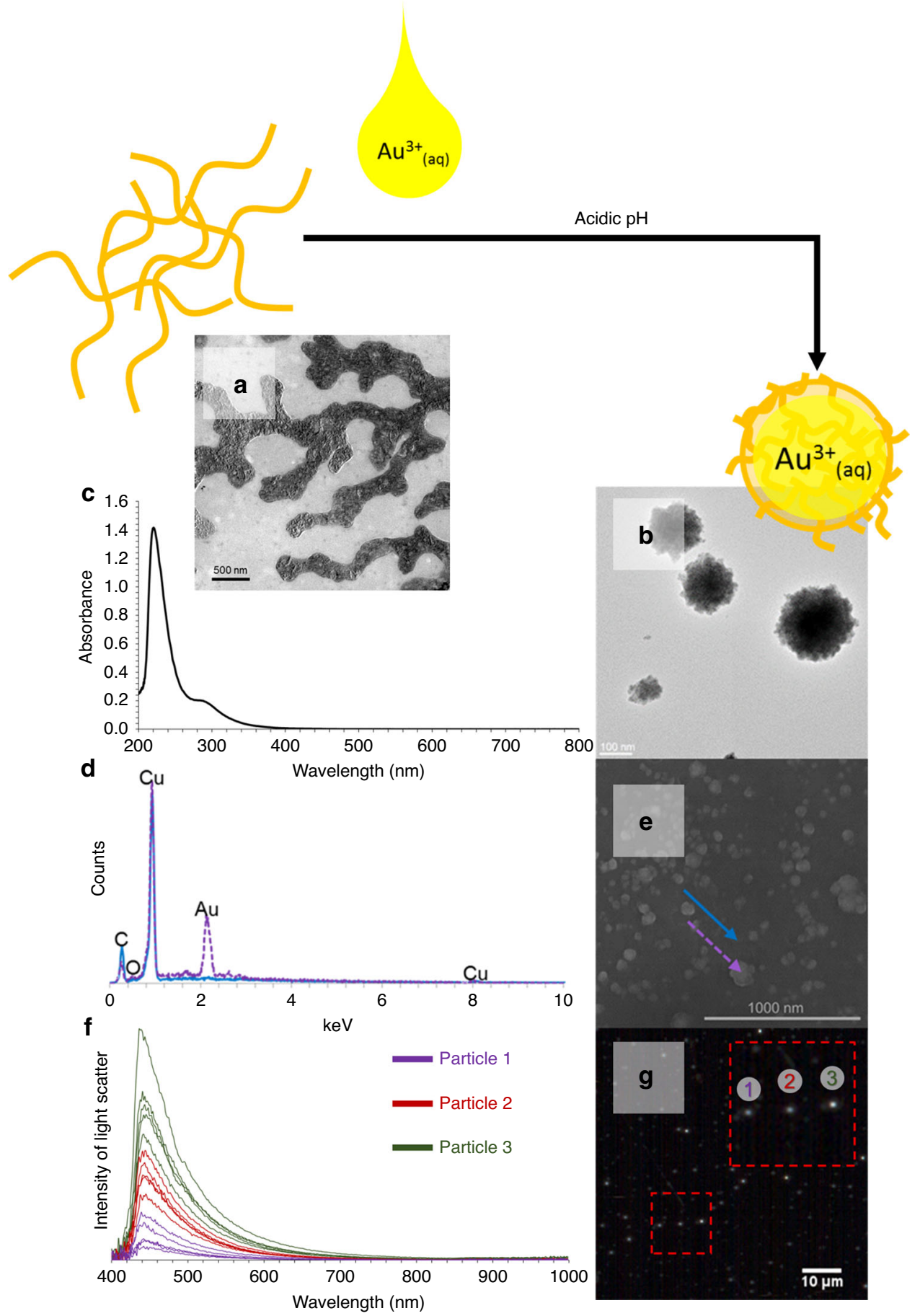

Fig. 1 Characterization of ionic Au-PEG delivery vehicle. Representative transmission electron microscopy (TEM) images of (a) PEG and (b) ionic AuPEG clusters. c UV-vis absorbance spectrum of ionic Au-PEG clusters (200-800 nm). d Electron dispersion spectrum of ionic-Au-PEG clusters (on copper tape) captured from (e) scanning electron microscopy-energy dispersive spectra (SEM-EDS). Purple spectra correspond to signal obtained from the clusters, where the blue spectra corresponds to the background. f Spectral intensity of light scatter obtained through enhanced dark field hyperspectral imaging of discrete Au-PEG clusters in solution ( $n=3$ particles, with 5 spectra from each). $\mathbf{g}$ Enhanced darkfield hyperspectral image with $2 X$ digital magnification where the red box highlights the particles those represent the spectra as shown in (f).

transmission electron microscopy (TEM) (Fig. 1b). From scanning electron microscopy-energy dispersive spectrum (SEM-EDS), we confirmed that the Au-PEG clusters contain gold (Fig. 1d, e) that is localized within the PEG clusters rather than dispersed within the solution. The dark contrast in TEM afforded by these clusters (Fig. 1b), as well as light scatter from dark field hyperspectral imaging of the clusters in solution (Fig. 1f, g) further supports these observations that the ionic gold is localized within the PEG clusters. The light scatter afforded through enhanced dark-field hyperspectral imaging for the non-reduced ionic gold particles confirmed the lack of surface plasmon resonance peaks that would correspond to the reduced gold. The light scattering peak at $420 \mathrm{~nm}$ is an artifact of the Vis-NIR liquid light guide which transmits light beginning at $420 \mathrm{~nm}$. Thus, using these spectral and microscopic techniques, we 

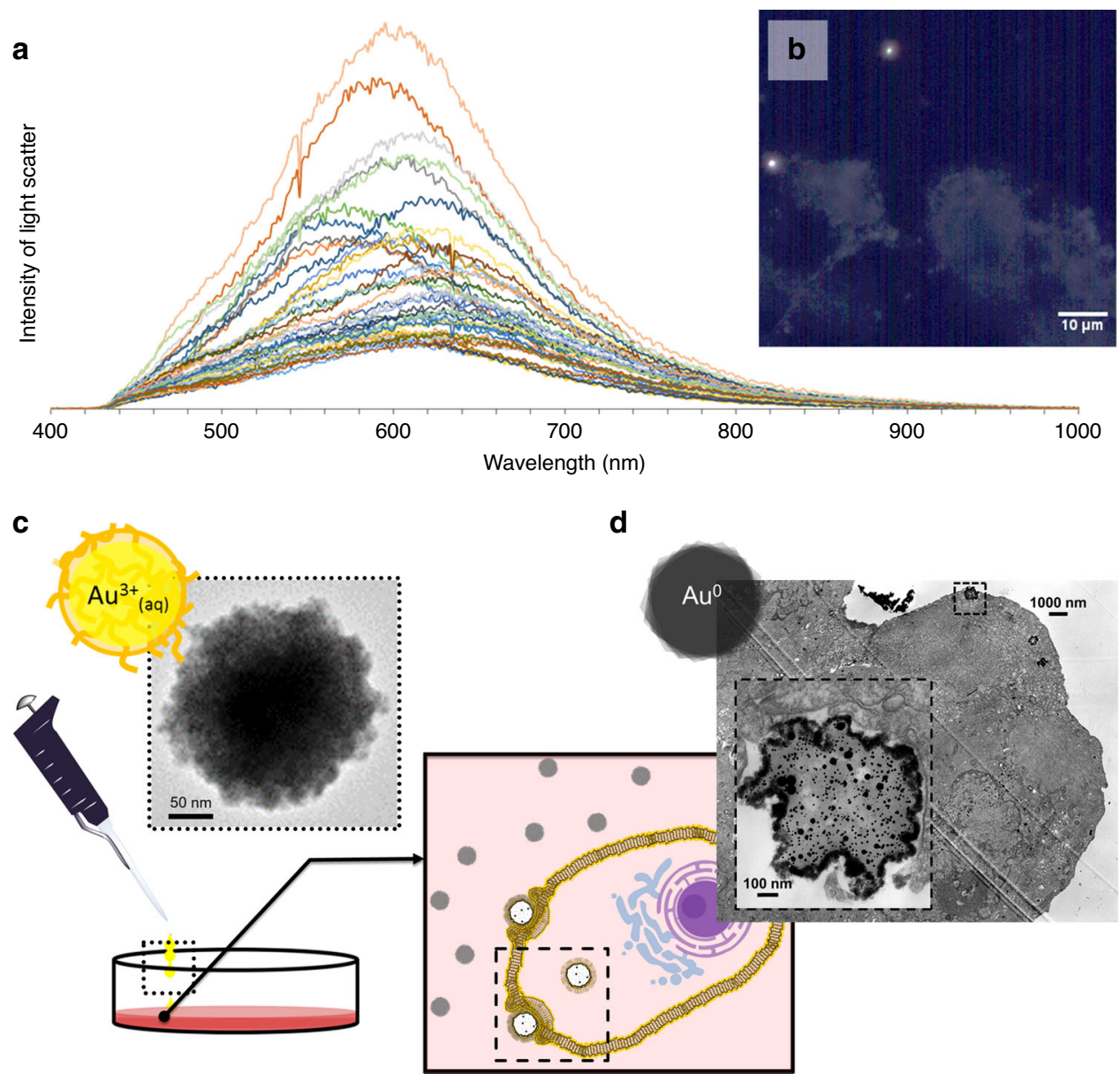

Fig. 2 Confirmation of in vitro reduction occurring en route. a Spectral intensity of light scatter from MCF-7 cells treated with Au-PEG clusters ( $n=44$ spectra), as distinct from untreated MCF-7 cells obtained through hyperspectral enhanced dark field imaging. $\mathbf{b}$ Corresponding enhanced hyperspectral darkfield image mapping of nanoparticles generated through Au-PEG treatment to MCF-7 cells with negative filtering with untreated MCF-7 cells (Supplemental Fig. 1e) to ensure no false-positive nanoparticle mapping. Scheme (c, d) with representative TEM images describing the reduction of ionic Au-PEG clusters (c), upon their interaction with cells (d).

have successfully characterized our delivery vehicles as containing ionic gold in discrete nanoscaled packets (without auto-reduction).

Forming gold nanoparticles via cellular mechanisms. Ionic $\mathrm{Au}-$ PEG clusters, prepared with $0.24 \mathrm{mM} \mathrm{Au}{ }^{3+}$, were capable of enabling a cellular reduction within $4 \mathrm{~h}$ of treatment in human breast cancer cell cultures (MCF-7) in complete cell media (containing 10\% FBS, with $1 \mathrm{x}$ penicillin and streptomycin). Previous applications for generating gold nanoparticles intracellularly, typically apply $1 \mathrm{mM}$ of ionic gold over time periods $>24 \mathrm{~h}^{36-47}$. However, the amount of formed plasmonic gold nanoparticles were negligible or considerably reduced when the ionic gold was applied diffusively in the previous literature reports either at lower concentrations $\left(0.1 \mathrm{mM}^{43,44}, 0.25 \mathrm{mM}^{42}\right)$ or for shorter time periods $\left(12 \mathrm{~h}^{42}, 15 \mathrm{~h}^{45}\right)$. From our application, we were able to confirm the transition of ionic to plasmonic gold through the spectral library obtained from the enhanced darkfield hyperspectral imaging of Au-PEG clusters reduced through the interactions with MCF-7 cells (Fig. 2a, b). Hyperspectral imaging technique was used to investigate the intracellular bioreduction process of ionic gold. It was found that although the treated precursor concentration was quite low than the previously reported ones, characteristic surface plasmon bands for $\mathrm{Au}(0)$ could only be observed after the bio-reduction process (Fig. 2a) in contrast the absence of plasmonic peaks for Au-PEG clusters (Fig. 1f). This further confirms that the bio-reduction process led to the formation of AuNPs with the oxidation state of $\mathrm{Au}(0)$ from the +3 state at Au-PEG ionic clusters. To confirm that these plasmonic nanoparticles were internal and not simply on the cell surface, TEM imaging of MCF-7 cells treated with Au-PEG clusters was performed (Fig. $2 \mathrm{c}$ and Supplementary Fig. 1d). An electron density focusing in formed particles with a dark, continuous, irregularly shaped border along the periphery was observed during this experiment (Fig. 2c). Further to confirm that gold nanoparticle formation is occurring en route to cellular internalization and not by undesired reactions with either the culture plate coating or the culture media, we undertook several control experiments. One such possibility is the presence of high number of primary amines on the adherent culture plate surface (i.e. poly-L-lysine coating) may induce reduction of ionic gold within the solution of the culture plate. But no reductive or structural changes were observed in this case even after incubating the ionic Au-PEG clusters for $24 \mathrm{~h}$ at $37^{\circ} \mathrm{C}$ on standard culture plates with poly-L-lysine coating (Supplementary Fig. 1b). Bovine serum albumin (BSA), a protein nutrient source within the culture media, is capable of reducing gold nanoparticle with the aid of mild to strong reductants, heating or $\mathrm{pH}$ manipulation (outside of physiological norms) ${ }^{48,49}$. We confirmed that BSA was not the source of gold biomineralization in our system, as the 
a

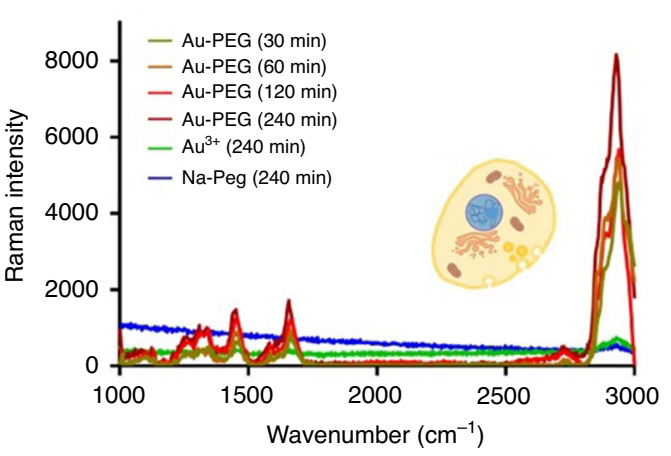

C

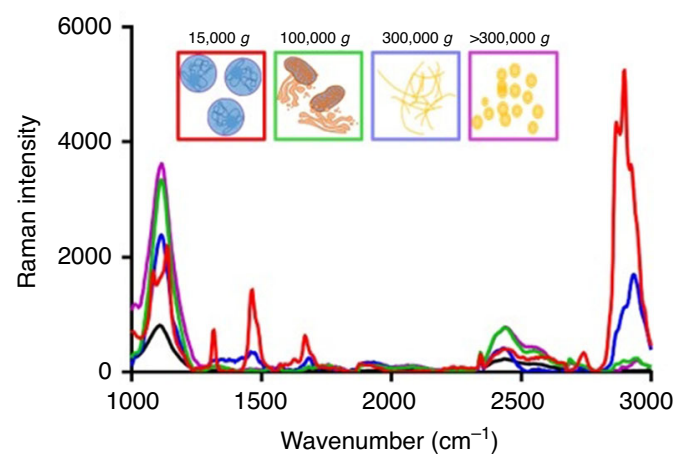

b

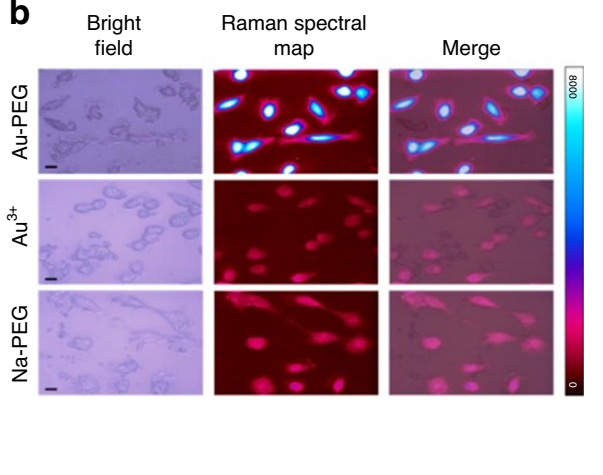

d

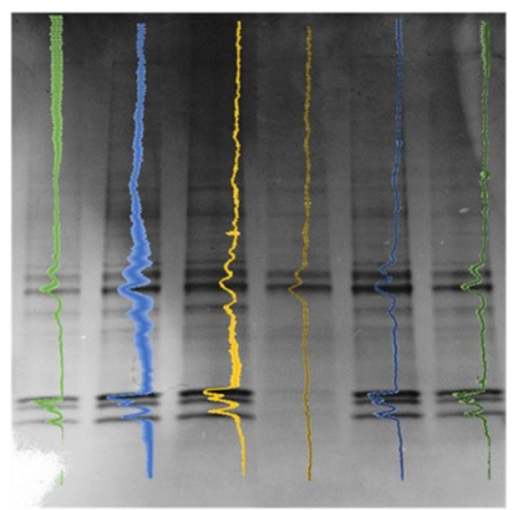

Fig. 3 Characterization of intracellular reduction of ionic gold. Raman spectra (a) of cells treated with either ionic Au-PEG clusters after 240 min (burgundy), $120 \mathrm{~min}$ (red), $60 \mathrm{~min}$ (orange), $30 \mathrm{~min}$ (yellow), $\mathrm{Au}^{3+}$ (green), or Na-PEG (blue). Raman mapping (of 2800-3000 peak intensity) with the corresponding bright field and merged images for 240 min treatments of Au-PEG, Au ${ }^{3+}$ and Na-PEG (b). Scale bars for (b) are $20 \mu \mathrm{m}$. c Raman spectra of Au-PEG-treated MCF-7 cellular fractions separated using differential centrifugation including the nucleus and large organelles, 15,000 $g$ (red);

mitochondria, lysosomes and other medium sized organelles, 100,000 g (green); membrane fragments, 300,000 g (blue); and highly soluble/cytosolic molecules (violet), >300,000 g; and the glass slide without cell fractions (black). d SDS-PAGE stained with Coomassie Blue with intensity plots overlaid of protein from the nuclear fraction of MCF-7 cells without treatment (green), treated with Na-PEG (blue), or Au-PEG (yellow) with standard SDS-PAGE conditions (lighter-shaded colors, left three lanes), or without $\beta$-mercaptoethanol (darker-shaded colors, right three lanes).

fresh culture media (containing BSA) was not able to reduce the ionic gold clusters $\left(24 \mathrm{~h}, 37^{\circ} \mathrm{C}\right)$ as confirmed through TEM images of this treatment solution (Supplementary Fig. 1c). For BSA-based biomineralization procedures, it is necessary to include strong/mild reductants or basic $\mathrm{pH}$ manipulation ${ }^{49,50}$. Contrary to these, neither strong/mild reductants, $\mathrm{pH}$ manipulation, or heating (beyond physiological norms) are present within the protocol of our system. Further the final $\mathrm{pH}$ of the cell surrounding was tested while $\mathrm{HAuCl}_{4}$ was added to the cell media for a final concentration of $1 \mathrm{mM}$, more than four times the concentration that was used in the current study. We observed that the change in $\mathrm{pH}$ was $<0.1$ suggesting the claim of benign environment for the biomineralization of gold nanoparticles. Thus, to confirm that our gold nanoparticle formation is enhanced directly as a result of our delivery vehicle, Raman micrographs of cells treated with Au-PEG clusters, chloroauric acid $\left(\mathrm{Au}^{3+}\right)$ (lacking $\mathrm{PEG}$ ), and a negative control containing PEG and sodium salt $\left(\mathrm{Na}^{+}\right)$in equal molar concentration of the analyte $\left(\mathrm{Au}^{3+}\right)$ were acquired. It was observed that the Raman intensity for the cells treated with Au-PEG were markedly enhanced $(8 \times)$ compared to cells treated with Na-PEG or just chloroauric acid only after four hour of incubation period (Fig. 3a, b). Additionally, this Au-PEG treatment begins to provide plasmonic enhancement as early as $30 \mathrm{~min}$ after treatment (Fig. 3a). This enhancement can only arise from species within a range of $<100 \mathrm{~nm}$. This plasmonic enhancement, only found from the cells treated with Au-PEG, confirmed the essential role of discretization of the $\mathrm{Au}^{3+}$ cations within a delivery vehicle for plasmonic nanoparticle formation at the applied concentrations and time frame.

Investigation on participating biomolecules. In order to utilize intracellularly formed GNPs directly for the biomedical applications (either diagnostic, therapeutic or a combination of both), knowledge of their formation process induced by the cellular biomolecules must be obtained. Elucidating the formation process and knowledge on the participating biomolecules for the formation of these gold nanosystems would provide valuable information relating to the possible pathological pathways with which this process could potentially be suitable for diagnostic/therapeutic applications. The enhanced Raman spectral maps afforded by ionic Au-PEG treatments to MCF-7 cells (Fig. 3a, b) provided important chemical information regarding the biomolecules proximal to the intracellularly formed nanoparticles. The biomolecules, proximal to the intracellularly formed nanoparticles, may be bound to or involved in process of their formation. The enhanced Raman spectra contain characteristic peaks arising from lipid (1175-1385, 1446-1477, and 2849-2969 $\left.\mathrm{cm}^{-1}\right)$, and proteins $\left(1590-1650 \mathrm{~cm}^{-1}\right)^{51}$. Additionally, lack of enhanced characteristic phosphate peaks at $2380-2450 \mathrm{~cm}^{-1}$ in the enhanced Raman spectra allowed us to confirm that phosphates from the sodium phosphate buffer and/or the nucleic acids are not participating in this gold reduction (Fig. $3 \mathrm{a})^{51}$. However, 
the presence of amide, $\mathrm{CH}_{3}$, and $\mathrm{CH}_{2}$ peaks do not provide enough information on their own to be considered viable as a diagnostic tool. In addition, this information does not prove precisely what physiologic processes are occurring within our system. To more precisely determine the biomolecules engaged in this intracellular reduction pathway, and which portions of the cell they originate from, cellular fractions of cells treated with the ionic gold nanoclusters were separated by density (through differential centrifugation) and the presence of plasmonic gold was subsequently investigated through acquisition of Raman spectra (SERS) (Fig. 3c). To generate these cellular fractions, mechanical lysis by tip sonication ( $30 \mathrm{~s}$ at $1 \mathrm{~A}$ ) of MCF-7 cells treated with the ionic Au-PEG or untreated cells was performed ${ }^{52}$. Then the fractions were separated by density via differential centrifugation into fractions typically associated with organelles of different sizes, i.e. (i) nuclei and large organelles, pellet from $15,000 \times g$ for $5 \mathrm{~min}$; (ii) mitochondria, lysosomes, peroxisomes, medium organelles, pellet from $100,000 \times g$ for $60 \mathrm{~min}$; (iii) cellular membrane fragments, pellet from $300,000 \times g$ for $120 \mathrm{~min}$; and (iv) highly soluble peptides/cytosolic molecules, supernatant from $300,000 \times \mathrm{g}$ for 120 $\min )^{53}$. For these fractions, corresponding Raman spectra were collected from cells either treated and or not treated with Au-PEG (Fig. 3c and Supplementary Fig. 2). Significant enhancement of the Raman spectra was observed for the fraction containing nuclei and the fraction containing cell membrane fragments from the cells treated with Au-PEG. Both the nuclear fraction and cellular membrane fragment fraction contained noticeable amide peaks at $1590-1650 \mathrm{~cm}^{-151}$. Due to the prominent amide peak, we were led to believe that these proteins were playing a significant role in this intracellular reduction process. The enhanced Raman spectra found in nuclear fraction, which implies localization of Au-PEG to the nucleus, does not necessarily imply internalization of the particles to the nucleus. Since the Raman microscope imaging is $2 \mathrm{D}$, we cannot make this assertion from the cell imaging either. However, since there is a distribution of nanoparticle sizes, from sub $5 \mathrm{~nm}$ to larger, it could be inferred that some smaller particles may pass through the nuclear pores $(\sim 6 \mathrm{~nm})$ to get internalized.

The notion, that proteins are involved in cellular biomineralization, has been explored by Singh et al. ${ }^{42}$, however, their system is resultant from diffuse applications of $\mathrm{Au}^{3+}$ so we should not infer that these systems/processes are identical. To determine if proteins are involved in our intracellular gold nanoparticle formation, a Coomassie Blue stained, sodium dodecyl sulfate-polyacrylamide gel electrophoresis (SDS-PAGE) was used to separate proteins found in the fractions from cells treated with Au-PEG, Na-PEG, or untreated (Fig. 3d and Supplementary Fig. 3a-d). We hypothesized that from Au-PEG and Na-PEG treatments, protein band intensity may be affected; and from the Au-PEG treatment, nanoparticle-bound proteins could be slowed in their electrophoretic migration through the gel due to the additional mass of gold. From this gel, we found no discernable difference between specific bands present nor their relative expression between treatments (Fig. 3d and Supplementary Fig. $3 \mathrm{a}-\mathrm{d}$ ). The information provided by this gel posed two possible major implications-(1) none of our treatments were affecting protein expression/integrity, and, all proteins which were running through the gel in detectable quantities did not adhere to the gold nanoparticles, and/or (2) proteins were not involved in this process. Another possible explanation, which we explored, was that one of the reagents used for preparing the protein for SDS-PAGE separation may have possibly interfered with the protein-gold interactions, effectively freeing the involved proteins from the nanoparticles. The primary candidate considered for this interference was $\beta$-mercaptoethanol $(\beta \mathrm{ME})$, as it is known to function as a highly efficient capping/quenching agent for gold nanoparticle syntheses capable of breaking $\mathrm{Au}-\mathrm{S}$ bonds between nanoparticles and protein ${ }^{54}$. To determine whether $\beta \mathrm{ME}$ was responsible for this freeing of Au-bound proteins, we were prompted to run an additional gel comparing protein migration from the fractions of cells either treated with Au-PEG, Na-PEG, or no treatment in duplicate-half would receive $\beta \mathrm{ME}$ and the other half would not (Fig. $3 \mathrm{~d}$ and Supplementary Fig. 3a-d). From these protein gels, we found distinct band intensity differential in the $10,000 \times g$ (nuclei and large organelle) protein fraction between the well with Au-PEG $\beta \mathrm{ME}(-)$ and all other wells of the same gel (Fig. $3 \mathrm{~d}$ and Supplementary Fig. 3a-d). The identical protein bands observed in control, Na-PEG, and Au-PEG of $\beta \mathrm{ME}$ containing lanes indicated that neither the Na-PEG nor the Au-PEG treatment affected the protein expression/integrity of proteins found within the nuclear fraction. Comparing this observation with the lanes of the gel that were lacking $\beta \mathrm{ME}$, we found that changes in protein band intensity were observed only in the Au-PEG lane. These changes in protein band intensity, which exclusively decreases in intensity, were only observed in the Au-PEG $\beta \mathrm{ME}$ lacking lane. This indicated that several proteins within that layer are attached to the formed plasmonic gold nanoparticles and that these proteins have maintained integrity. Having observed protein impedance within the nuclear fraction of the ionic Au-PEG treated cells, we aimed to identify these proteins through LC-MS protein fingerprinting.

In order to identify proteins involved in intracellular gold nanoparticle formation through mass spectroscopy, we separated involved proteins from those not involved through gel electrophoresis. From our previous Coomassie-stained gel experiments, we found that involved proteins would remain bound to the gold nanoparticles preventing their electrophoretic migration through the gel. To separate these gold bound proteins from the noninvolved proteins, we would run the nuclear fraction of ionic AuPEG treated cells without $\beta \mathrm{ME}$ for an excess of time $(>3 \mathrm{~h})$, at which point the largest ladder protein would have run off the gel $(250 \mathrm{kDa})$ and we would then separate the top portion of the gel containing the wells $(1.5 \mathrm{~cm}$ ) from the bulk (Supplementary Fig. 4). The proteins/gold particles, located within either of these gel portions, were then extracted using a diffusion-mediated passive elution method ${ }^{55-57}$. These extracted proteins were then briefly trypsinized to fragment the proteins present from extractions and centrifuged at $6000 \times g$ to pellet any gold nanoparticles out of the solution. The supernatant containing soluble protein fragments was then analyzed via LC-MS with MASCOT analysis (Matrixscience, Boston, MA). We found almost 2239 different protein fragments within the top portion of the gel, which contained proteins that were most impeded through the gel. These protein fragments identified within the top portion of the gel, including only those with a protein score $>21$ $(\alpha<0.05)$ and uniquely found in the top of the gel (excluding large slow moving proteins found in the bulk portion), accounted for 16 different proteins (Supplementary Tables 1, 2). These proteins are located, as annotated on UniProt.org through COMPARTMENTS ${ }^{58}$, include subcellular regions ranging from extracellular to the nucleus (Supplementary Table 1). These proteins of interest are involved in 287 different molecular processes as identified through phylogenetic-based propagation of functional gene ontology (GO) annotations (Supplementary Table 2$)^{59}$. Of these molecular processes, the most conserved GO term processes are related to binding (Table 1). Eleven of the 16 identified proteins are involved in binding processes, eight of those in protein binding, five in metal/calcium/cationic binding, three in ATP/nucleic acid binding, two in antigen binding, two which directly bind with each other, and two which bind actin filament (Table 1). These 11 proteins, involved in molecular binding and the intracellular reduction of gold nanoparticles, are 
Table 1 Proteins adhered to intracellularly formed gold nanoparticles identified through LC-MS of the nuclear fraction of MCF-7 cells treated with ionic Au-PEG clusters.

\begin{tabular}{|c|c|c|c|c|c|c|c|c|c|c|c|c|}
\hline & $\begin{array}{l}\text { Protein } \\
\text { name }\end{array}$ & $\begin{array}{c}\text { Alpha } \\
\text { amylase } \\
2 \mathrm{~B}\end{array}$ & $\begin{array}{c}1,4- \\
\text { alpha- } \\
\text { glucan- } \\
\text { branching } \\
\text { enzyme }\end{array}$ & $\begin{array}{l}\text { Immunoglobu } \\
\text { lin lambda- } \\
\text { like } \\
\text { polypeptide } 5\end{array}$ & $\begin{array}{c}\text { Immunoglobulin } \\
\text { heavy } \\
\text { constant } \\
\text { alpha 1 }\end{array}$ & $\begin{array}{l}\text { Cadheri } \\
n-23\end{array}$ & $\begin{array}{c}\text { SRC } \\
\text { kinase } \\
\text { signaling } \\
\text { inhibitor 1 }\end{array}$ & $\begin{array}{l}\text { Integrin } \\
\text { beta-1- } \\
\text { binding } \\
\text { protein } 1\end{array}$ & $\begin{array}{c}\text { Calmoduli } \\
n-1\end{array}$ & Titin & $\begin{array}{c}\text { Protein } \\
\text { FRG1 }\end{array}$ & $\begin{array}{l}\text { Probable } \\
\text { ATP- } \\
\text { dependent } \\
\text { RNA } \\
\text { helicase }\end{array}$ \\
\hline & Gene & AMY2B & GBE1 & IGLL5 & IGHA1 & $\mathrm{CDH} 23$ & SRCN1 & ITGB1BP1 & CALM1 & TTN & FRG1 & DDX31 \\
\hline \multirow{4}{*}{ 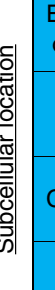 } & $\begin{array}{l}\text { Extracellular } \\
\text { or secreted }\end{array}$ & & & & & & & & & & & \\
\hline & $\begin{array}{l}\text { Plasma } \\
\text { membrane }\end{array}$ & & & & & & & & & & & \\
\hline & Cytoskeleton & & & & & & & & & & & \\
\hline & Nucleus & & & & & & & & & & & \\
\hline \multirow{14}{*}{ है| } & $\begin{array}{l}\text { Protein } \\
\text { binding }\end{array}$ & & & & & & & & & & & \\
\hline & $\begin{array}{l}\text { Protein } \\
\text { kinase } \\
\text { binding }\end{array}$ & & & & & & & & & & & \\
\hline & $\begin{array}{l}\text { Protein } \\
\text { domain } \\
\text { specific } \\
\text { binding }\end{array}$ & & & & & & & & & & & \\
\hline & $\begin{array}{l}\text { Cation } \\
\text { binding }\end{array}$ & & & & & & & & & & & \\
\hline & $\begin{array}{l}\text { Metal ion } \\
\text { binding }\end{array}$ & & & & & & & & & & & \\
\hline & $\begin{array}{l}\text { Calcium ion } \\
\text { binding }\end{array}$ & & & & & & & & & & & \\
\hline & $\begin{array}{l}\text { Antigen } \\
\text { binding }\end{array}$ & & & & & & & & & & & \\
\hline & $\begin{array}{l}\text { Immunoglob } \\
\text { in receptor } \\
\text { binding }\end{array}$ & & & & & & & & & & & \\
\hline & Titin binding & & & & & & & & & & & \\
\hline & $\begin{array}{l}\text { Calmodulin } \\
\text { binding }\end{array}$ & & & & & & & & & & & \\
\hline & $\begin{array}{l}\text { Actin } \\
\text { filament } \\
\text { binding }\end{array}$ & & & & & & & & & & & \\
\hline & ATP binding & & & & & & & & & & & \\
\hline & $\begin{array}{l}\text { Nucleotide } \\
\text { binding }\end{array}$ & & & & & & & & & & & \\
\hline & $\begin{array}{l}\text { RNA } \\
\text { binding }\end{array}$ & & & & & & & & & & & \\
\hline
\end{tabular}

characteristically located in regions ranging from extracellular to the nucleus ${ }^{58}$. Since our extraction and isolation of these goldbound proteins was from the nuclear fraction and many of these proteins are not typically located in the nucleus, this suggests that progressive intracellular reduction may occur and continue sequentially, accumulating a protein corona from multiple regions of the cell (Fig. 4). This proposed migratory path with sequential reduction towards the nucleus initiates as the ionic gold delivery vehicle interacts with the secreted proteins (Fig. 4a). To simulate this primary reduction process, which occurs extracellularly, we admixed ionic Au-PEG clusters with spent cell media (cells have been incubated with media for $\sim 24 \mathrm{~h}$ ). Through TEM imaging we were able to find electron density focusing within the PEG clusters potentially indicating 


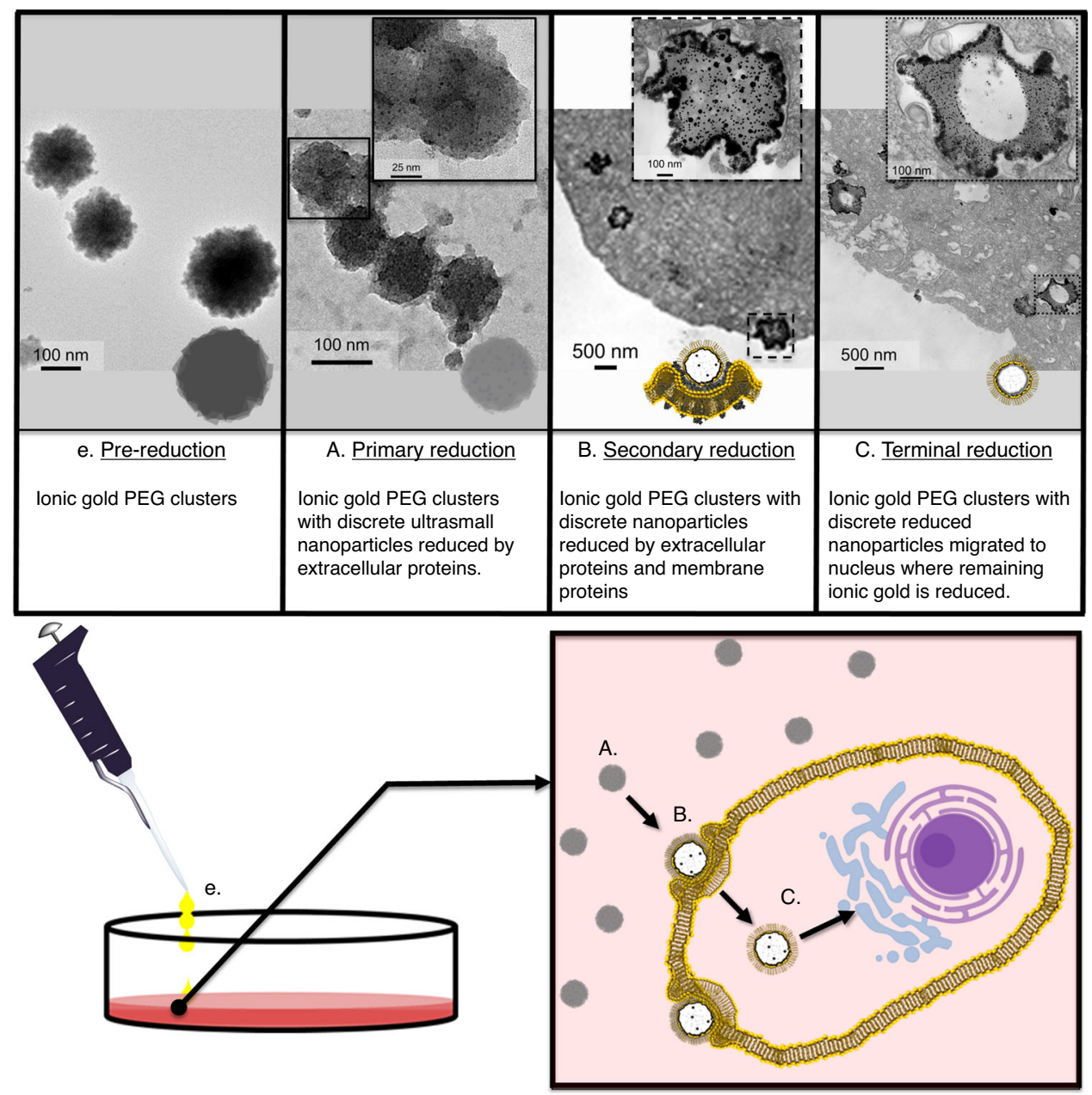

Fig. 4 Scheme of progressive reduction process. Pictorial diagram for the intracellular formation of gold nanoparticles via Au-PEG clusters throughout their migration to the nucleus with TEM images of respective reduction phases.

nanoparticle formation (Fig. 4a). This electron density focusing is not observed when ionic Au-PEG clusters are admixed with fresh cell media (Supplementary Fig. 1b), indicating that this electron density focusing is resultant of cellular biomolecule secretions. The proteins involved in this primary reduction include those involved in antigen binding/phagocytosis (IGLL5, IGHA1), metabolic/ catalytic activity (ZG16B, AMY2B, GBE1), cation binding (AMY2B and GBE1), and steroid binding (SCGB1D2) ${ }^{59}$. Two of these proteins involved in primary reduction are also located in multiple cellular regions (GBE1 and IGHA1) posing as potential migration enablers to the other cellular regions ${ }^{58}$. In addition, their localization to the extracellular regions, GBE1 and IGHA1 are also located within the cytoplasm and plasma membrane regions, respectively ${ }^{58}$. This potential for mitigating migration is especially notable for IGHA1, as this protein is both located in the plasma membrane and involved in receptor-mediated phagocytosis. The proteins GBE1 and IGHA1 could act as enablers for migration of the ionic Au-PEG clusters from the primary to secondary reduction phase, wherein the clusters interact with the plasma membrane (Fig. 4b). Through cellular TEM images of our ionic gold PEG clusters, we were able to see additional electron density focusing compared with that found via interactions with cell secretions as they interact with the protein-rich membrane (Fig. 4b). The additional electron density focusing shows nanoparticles within the clusters as more polydisperse than from the primary reduction with the addition of a dark electron-dense border contiguous with the plasma membrane. Additionally, from enhanced Raman signals found in the membrane of whole cells (Fig. 3a, b) and in membrane fractions (Fig. 3c), we concluded that plasmonic nanoparticles exist in the plasma membrane. This secondary reduction, catalyzed by the proteinrich membrane, may be initiated by IGHA1 as it found extracellularly, within the plasma membrane, and involved in receptor-mediated endocytosis. As this endocytosis is initiated, the ionic Au-PEG cluster is proximal to the plasma membrane, this proximity subjects the clusters to higher concentrations of cellular proteins/biomolecules, which are either secreted or located within the plasma membrane providing an environment more conducive to nanoparticle formation. The proteins involved in this secondary reduction within the plasma membrane are involved in cation binding (IGHA1, CDH23), antigen binding (IGHA1), kinase regulation/binding (SRCN1, ITGB1BP1), protein transport (ITGB1BP1), receptor clustering (ITGB1BP1), and endocytosis (IGHA1) ${ }^{59}$. In addition to IGHA1, SRCN1, and ITGB1BP1 are also found in multiple cellular regions as potential migratory enablers. Moreover being located within the plasma membrane, SRCN1 and ITGB1BP1 are both located within the cytoskeleton and ITGB1BP1 is located within the nucleus ${ }^{58}$. Once internalization of the ionic Au-PEG clusters is complete, we observed through TEM that the electron density at the center of these clusters diminishes (Fig. 4c). In addition to the hollowing of the Au-PEG clusters, the electron-dense border is less defined compared to the secondary reduction phase (during interaction with the plasma membrane). A possible explanation for this 
reduction in electron density, is that, as the Au-PEG clusters interact with the plasma membrane, some portion of the gold may remain within the plasma membrane after the Au-PEG clusters are internalized. It is worthy to mention here that it is a diffusive regime-driven process. Once Au-PEG clusters reach the cell membrane, they get spontaneously reduced close to membrane landmarks and makes bigger clusters via spontaneous aggregation which limits their further diffusivity, hence some of the reduced $\mathrm{Au}$ remain distributed around cell periphery. Evidence supporting this explanation that some portion of the gold may remain within the plasma membrane, is found in the enhanced Raman spectra. Both in whole cells (Fig. 3a, b) and the extracted cell fractions (Fig. 3c) of those cells treated with ionic Au-PEG clusters showed enhanced Raman signal in the nucleus and membrane. This Raman enhancement indicated that nanoparticles resided within the plasma membrane and the nucleus after the $4 \mathrm{~h}$ of treatment period. The concept that gold nanoparticles remain within the plasma membrane as the ionic Au-PEG clusters continue to migrate toward the nucleus is supported by the reduction of overall electron density observed as these clusters are fully internalized. After internalization through the plasma membrane the Au-PEG clusters undergo progressive reduction, collecting proteins from the cytoskeleton (SRCN1, ITGB1BP1, CALM1), cytosol (PRDX4, GBE1), endoplasmic reticulum (PRDX4, JKAMP), and lysosome (GM2A) regions. The proteins in these non-nuclear regions are involved in metabolic processes (GM2A, CALM1), response to protein unfolding and oxidative stress (JKAMP, PRDX4), cation binding (CALM1, ITG1BP1), intracellular signal transduction (ITGB1BP1), and Titin binding (CALM1) ${ }^{59}$. The protein ITG1BP1, is involved in intercellular signal transduction and cation binding, and is located within the plasma membrane, cytoskeleton, and nucleus ${ }^{58}$. The protein CALM1, involved in cation binding and titin binding (a nuclear protein $)^{58}$. Both ITG1BP1 and CALM1 pose as likely potential moderators for migration of ionic Au-PEG clusters to the nucleus for terminal reduction because of their involvement in cation binding complementary with their subcellular locations and nuclear protein interactions. If ITGB1BP1 and CALM1 retain their normal protein function wherein they transduce signals to the nucleus through binding (while they are bound to the Au-PEG protein complex), they pose as a potential route for mediating the migration of the ionic Au-PEG clusters to the nucleus for terminal reduction. We ascertain that the ionic Au-PEG clusters migrate to the nucleus for terminal reduction because of the enhancement of the Raman (whole cell mapping and fractions) combined with the identification of proteins bound to gold that are exclusively found in the nucleus (DDX31, FRG1, TTN). These proteins, found exclusively within the nucleus, are involved in cation/calmodulin binding (TTN), actin filament binding (TTN, FRG1), ATP binding (TTN, DDX31), and nucleotide binding (DDX31, FRG1, TTN $)^{59}$. The protein titin $^{60}$, with at least 23 cation-binding sites as well as binding calmodulin, poses as a potential gateway for the ionic Au-PEG clusters' entry into the nucleus and then to the proximity of other nucleotide binding proteins (DDX31, FRG1). This complete intracellular progressive reduction process, involving at least 16 different proteins localized throughout the cell without apparent protein degradation (Fig. $3 \mathrm{~d}$ and Supplementary Fig. 3a-c), involving 287 different molecular processes (including nucleotide binding) with terminal reduction within the nucleus, poses as an interesting and innovative theranostic agent. Overall, the sequential bio-reduction process can be summerized as- (i) The first phase starts at extracellular regions where the proteins, IGLL5, IGHA1, ZG16B, AMY2B, GBE1, AMY2B, GBE1, and SCGB1D2, take part, where IGHA1 has been observed to be one of the prime protein responsible for the receptor-mediated phagocytosis of the Au-PEG clusters; (ii) The second phase starts within the plasma membrane where the proteins, IGHA1, CDH23, SRCN1, and ITGB1BP1 have taken part; (iii) Au-PEG clusters then undergo progressive reduction, collecting proteins from the cytoskeleton (SRCN1, ITGB1BP1, CALM1), cytosol (PRDX4, GBE1), endoplasmic reticulum (PRDX4, JKAMP), and lysosome (GM2A) regions and (iv) The migration of ionic Au-PEG clusters to the nucleus for terminal reduction occurs through the involvement of ITG1BP1 and CALM1. We have also ascertained DDX31, FRG1, TTN as the gold bound proteins those are exclusively found in the nucleus. This application, wherein nanoparticles are generated intracellularly through ionic Au-PEG clusters, would provide no plasmonic signal unless the treatment was successfully applied. From this $\mathrm{Au}-\mathrm{PEG}$ application, gold nanoparticles generated intercellularly are bound to intact proteins involved in almost 300 different molecular processes (including nucleotide binding), and resulting nanoparticles localized within the nucleus. It may be presumed that protein corona would form as soon as the NPs were introduced into the biological milieu. Photothermal therapy can therefore potentially be applied using this process which would disrupt a host of molecular processes simultaneously as well as directly cause heating of the nucleus, nuclear proteins, and essentially nucleotides.

To identify specific amino acids preferred in ionic gold reduction from these 16 proteins, we calculated the amino acid distribution in terms of overall percent for on-site protein fragments and off-site protein fragments and compared against the amino acid distribution of the native protein BLAST data from Uniprot. Further to identify which amino acids, in the identified proteins of interest, were preferentially interacting with and reducing gold, we made an assumption that amino acids which participate actively/directly in reduction would be bonded to the gold nanoparticles and in turn would be underrepresented in the LC-MS fragmentation analysis compared with the native AA distribution (Fig. 5). Comparing the change in distribution in AA representation in on-site proteins between fragment from LC-MS and whole native proteins (from Uniprot), we were able to determine which AAs were highly represented in the identified protein fragments (unlikely for participation in gold reduction [Lys]; [Arg]; [Pro]), and which were represented in fewer instances than in the native protein (likely candidate AAs are [Leu]; [Thr]). These bound amino acids would not appear in as high frequency as the uninvolved AAs through LC-MS analysis due to the random nature of the fragmentation combined with the gravimetric separation of protein fragments from dense gold nanoparticles. However, this AA distribution change (Fig. 5) does not account for the natural bias of the fragmentation process for LC-MS analysis. To account for the bias of the fragmentation process for LC-MS analysis, we would subtract the change in AA representation identified between fragments and whole native proteins identified off-site (bulk of the gel). Amino acids, which have a positive change in AA representation between fragments and whole native proteins identified off-site are considered overrepresented ([Arg] ) by the fragmentation process and those with a negative change considered to be underrepresented ([Cys]). To correct for the bias of the fragmentation process, we would subtract this bias/favorability in AA representation. Now comparing the distribution change in AA representation in on-site proteins after subtracting bias from the fragmentation process, we were able to determine more reasonably [Lys], [Arg], and [Pro] as unlikely candidates for reduction of gold and [Leu] and [Thr] as likely candidates (Fig. 5). However, this AA distribution change with subtraction bias of the fragmentation process does not account for the variability of AAs between onsite and off-site proteins (Fig. 5). From comparing the AA 

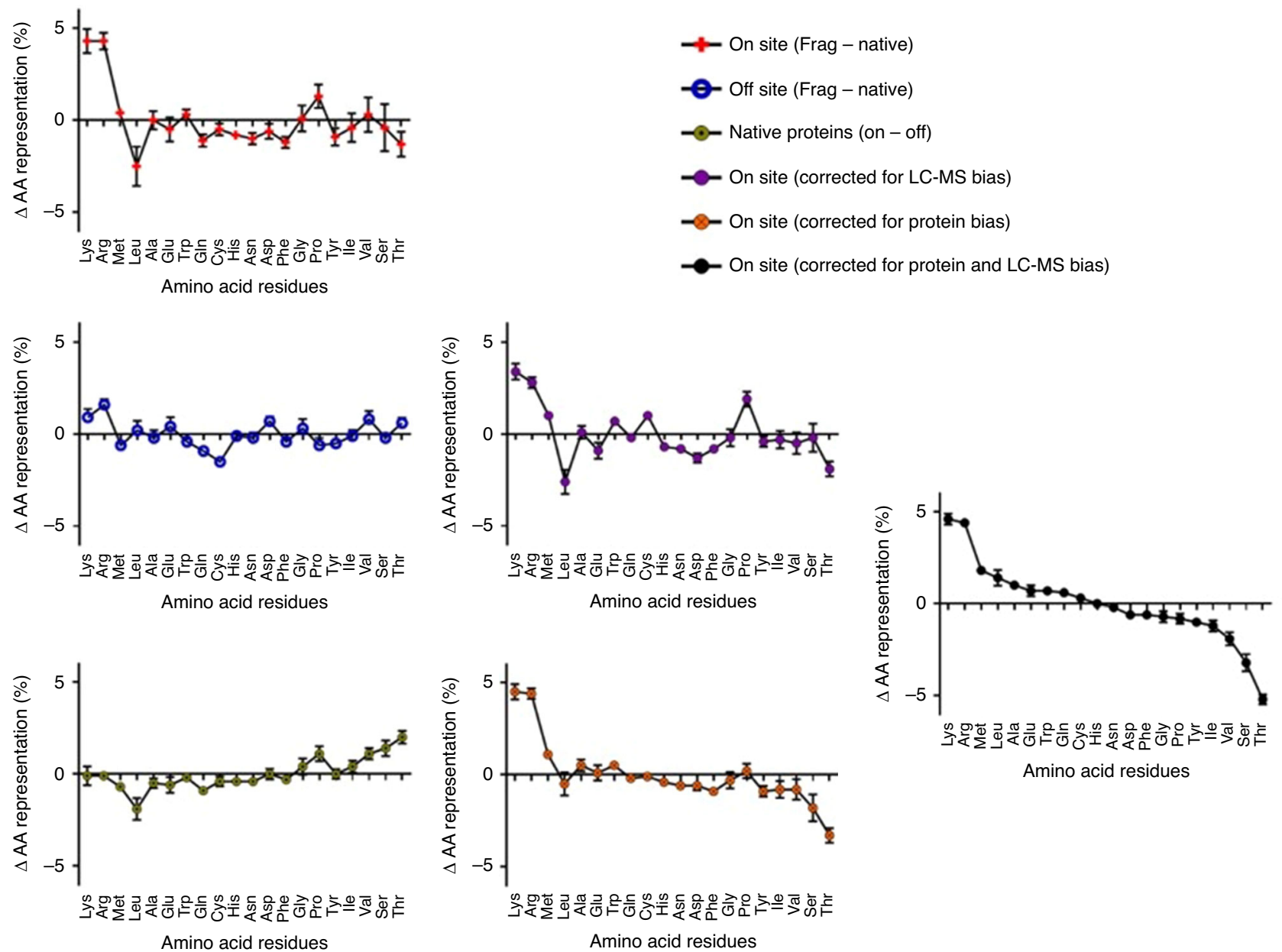

Fig. 5 Changes in amino acid distributions from proteins identified through LC-MS. Amino acid distributions for on-site proteins between those found in LC-MS fragments and in nature (red); for off-site proteins between those found in LC-MS fragments and in nature (blue) representing the bias of the trypsin fragmentation and the LC-MS-MASCOT system; for native proteins between those found on- and off-site (yellow) representing the preference of the amino acid distribution between on- and off-site proteins; and various correction subtractions (purple or orange are partial corrections and black includes all corrections). Error bars are standard deviations of the mean ( $n \geq 16$ proteins).

distribution between on-site and off-site proteins in their native states, we identified AAs which more highly represented in the target than off-site proteins ([Pro]; [Val]; [Ser]; [Thr]) and AAs which had lower representation ([Leu]). After subtracting this bias of the AA distribution between native states of the on-site and off-site proteins, we were able to identify [Lys], and [Arg] as overrepresented (likely not involved in reduction), and [Ser] and [Thr] as underrepresented (likely candidates for gold reduction).

In vitro impact. To characterize the cytotoxicity of our ionic gold delivery platform, we compared apparent metabolic impacts (through MTT) and physical morphological changes (through flow cytometry without staining) against the treatments with ionic gold (without PEG), PEG with ionic sodium, and Nifuroxazide with PEG (NIFU-PEG). From the MTT assay, our intracellular gold nanoparticle generating solution (Au-PEG) appears to reduce cell viability at lower treatment concentrations compared with $\mathrm{Au}^{3+}$ and Na-PEG with a treatment-response dependent more on concentration than NIFU-PEG (Fig. 6a). However, there is an apparent inflection point in the apparent viability of Au-PEG-treated cells which causes us to question reliability of this assay for our treatment. This inflection point occurs in MCF-7 cells as the apparent cell viability approaches $0 \%$. We do not believe that this inflection point in apparent cell viability reflects an actual resurgence in cell viability or a change in treatment behavior at higher concentration, but actually reflects a reaction between unreacted treatments (ionic gold) and the dye, Thiazolyl Blue Tetrazolium bromide. To support this hypothesis, that the unreacted ionic gold can react with the Thiazolium Blue, we incubated our cell treatments $\left(\mathrm{Au}^{3+}, \mathrm{Au}-\right.$ PEG, and Na-PEG) at titrated concentrations with Thiazolium Blue (at MTT concentrations) and measure absorbance at the same wavelength used for the MTT assay $(570 \mathrm{~nm})$. From incubating our cell treatments with Thiazolium Blue in the absence of cells, we found a linear relationship between the concentration of ionic gold and the absorbance used to measure cell viability through MTT (Fig. 6b). From this we can ascertain that the inflection point in the cell viability is due to unreacted ionic gold interacting with the Thiazolium Blue. In addition to this, if our treatment can potentially interact with dyes, dye-based assays may not be ideal for characterizing the impact. On the other hand, by running flow cytometry and collecting forward scatter vs. side scatter measurements, we can comment on the morphological changes undergone by the cells in presence of various treatments without the use of dyes. From forward scatter parameter, one can monitor the change in cellular size, whereas from the side scatter parameter, one can comment on the internal complexity of cells. As shown in Fig. 6c, we observe three distinct populations/regions, marked as I (dead cells), II and III (cellular 
a

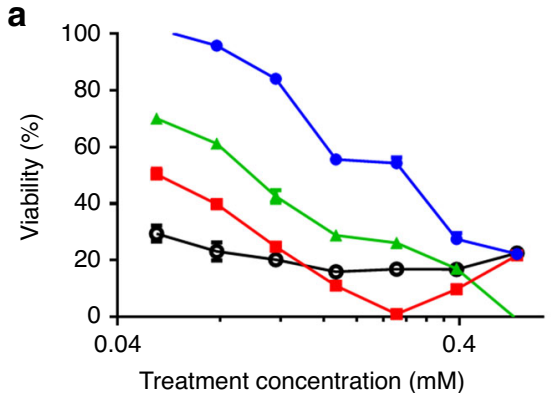

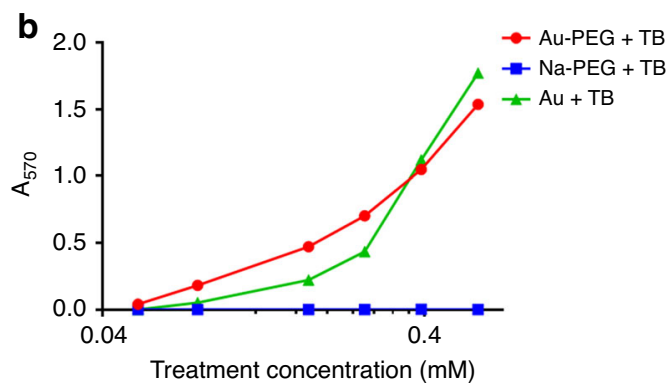
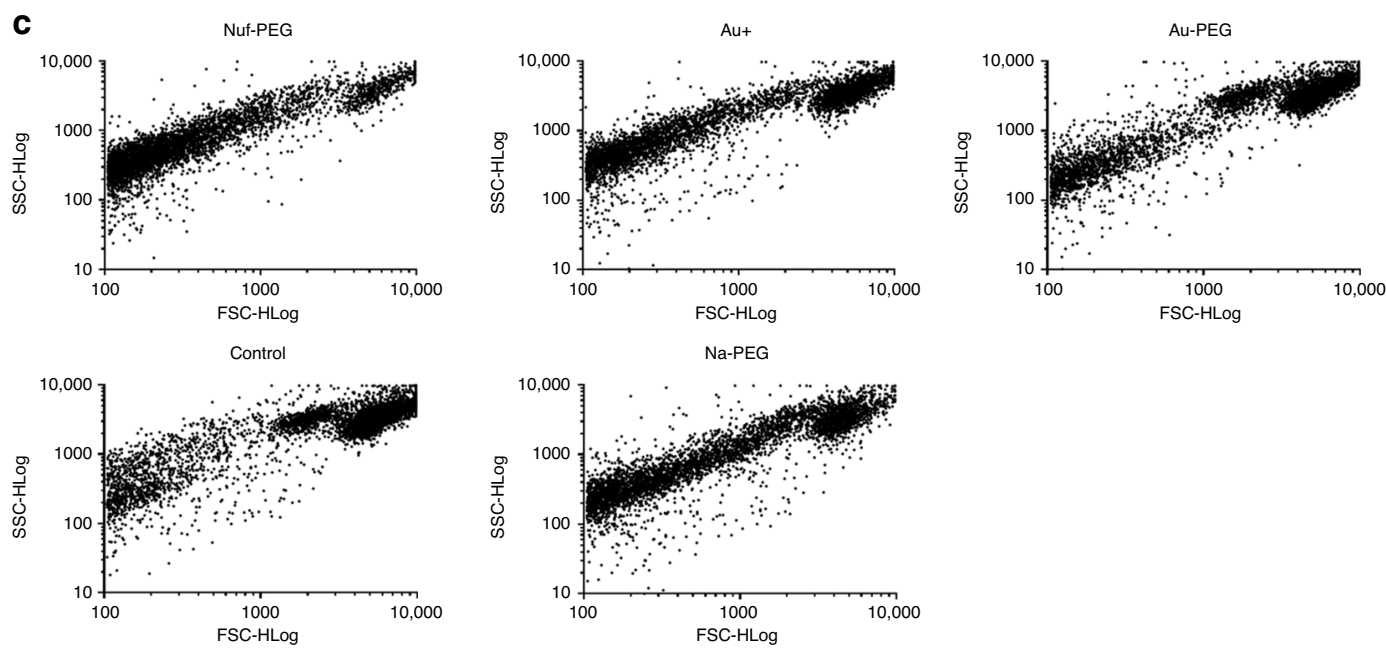

d
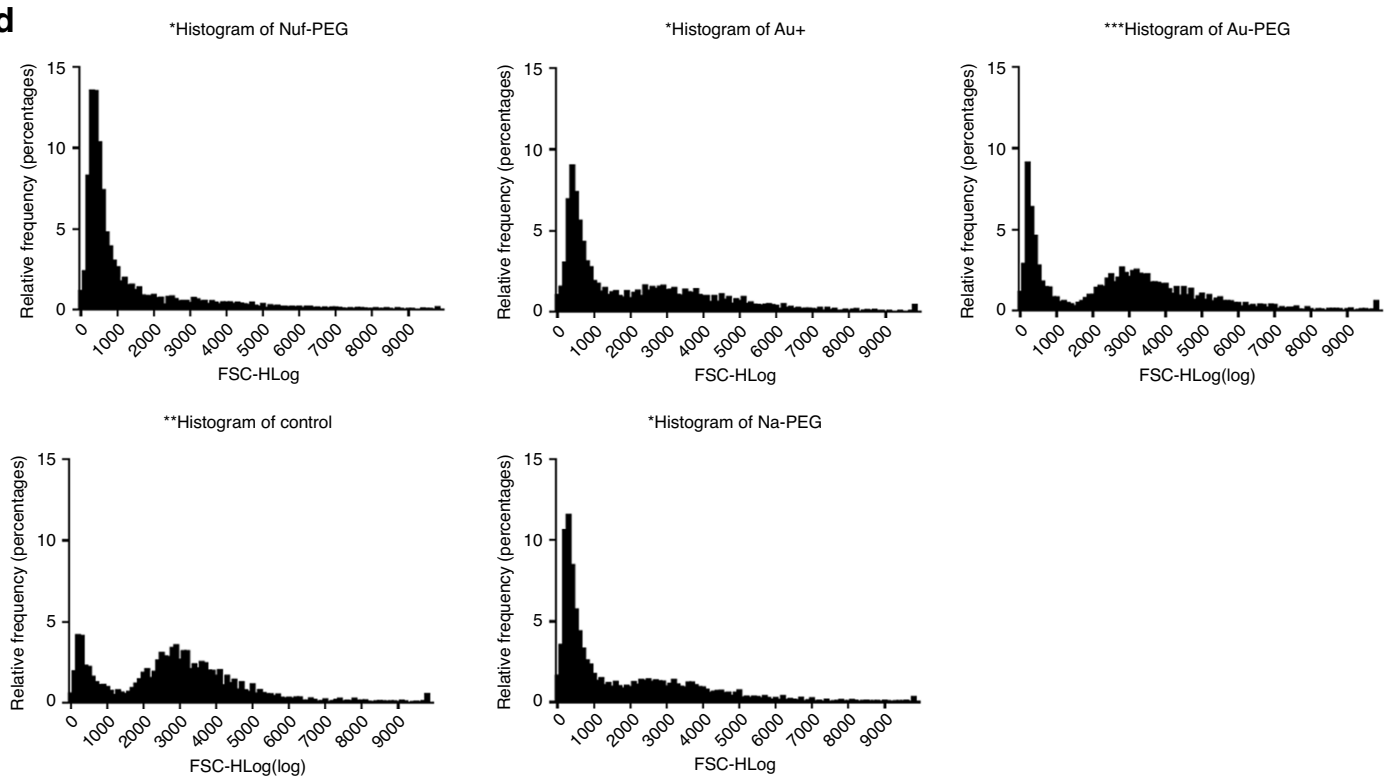

Fig. 6 Cell viability of MCF-7 when treated with ionic gold. a Cells as assessed via thiazolium blue (MTT) metabolic assay for cells treated with Na-PEG (blue), $\mathrm{Au}^{3+}$ (green), Au-PEG (red) and nifuroxazide mixed with PEG (black). For (a) and (b), error bars are standard deviation of the mean ( $n=3$ biologically independent samples). Titrated light interactions between ionic-PEG mixtures (same color scheme) as admixed with thiazolium blue at concentrations used for MTT assay. Comparison of forward scatter vs side scatter plot for different samples at $0.24 \mathrm{mM}$ treatments (c). Representative histogram analysis of different samples (d) showing two populations, one corresponding to dead cells at low FSC, whereas the second at higher FSC corresponding to healthy cells. Error bars in (a) and (b) are standard deviations of the mean.

components) for all the samples including control. However, treatment with NIFU-PEG showed a marked increase of population of region 1 (dead cells) when compared with control, which could be directly attributed to the initiation of apoptosis by nifuroxazide resulting in cell death. Further histogram analysis via relative frequency vs. forward scatter (Fig. 6d) supplements this finding, we obtained two populations, the first one corresponding to dead cells having low diameter, whereas the second population bigger in diameter and corresponding to healthy cells. For control and Au-PEG treatments, we observed a smaller number of dead cells and a greater number of healthy cells. However, for NIFU-PEG, most of the population had smaller 
diameter corresponding to dead cells, and minimal population at the higher diameter (healthy cells) owing to the toxic nature of nifuroxazide.

A microarray analysis was used to identify changes in gene expression for pathways related to apoptosis (Supplementary Fig. 5) with directed focus on expression changes at the end of these pathways for pro-survival or pro-apoptosis. From this we found upregulation of PMAIP1, MCL1, DDIT3 (pro-apoptosis) together inhibiting the pro-survival gene BCL2 (reacts to calcium and iron) for Au-PEG treatments when compared with untreated MCF-7 cells. Additionally, the GADD45 (pro-survival) gene was highly upregulated (associated with DNA damage and growth arrest) for Au-PEG treatments.

Investigation of internalization mechanisms. Cell internalization efficiency of our nanoparticles must be determined, so that we would be able to practically deliver high enough concentrations of gold for practical imaging purposes (X-Ray, photoacoustic, or fluorescence), and/or therapeutic photothermal ablation. To calculate the internalization efficiency, we treated MCF-7 cells in triplicate for $4 \mathrm{~h}$ with our ionic gold PEG clusters, collected the supernatant and cell pellet, submitted both for ICP analysis for gold and used the total mass of gold in the cell pellet vs. the supernatant to calculate the total internalized gold based on the treatment concentration. We found that nearly $50 \%$ of the gold was internalized in the breast cancer cells (Supplementary Fig. 6). We then investigated the internalization pathways those were involved using a series of inhibitors that would selectively block major endocytosis pathways (Supplementary Fig. 6). Small molecule inhibitors for our study selected in such a way that they did not exert any considerable toxicity by themselves in the short period of incubation. Furthermore, they were chosen so that they do not affect the actin cytoskeleton post treatment ${ }^{23,61}$. The working hypothesis of this study was based on the fact that a particular inhibitor would block a specific endocytic pathway and consequently not allow the nanoparticles to internalize in the cells via that particular pathway. A combination of 2-deoxyglucose (DOG) and sodium azide $\left(\mathrm{NaN}_{3}\right)$ were employed to inhibit glycogenosis and cellular respiration, respectively, via energydependent uptake ${ }^{62}$. Chlorpromazine (CPM) is known to reduce the generation of clathrin-coated pits via a reversible translocation of clathrin and its adapter proteins from plasma membrane to intracellular vesicles. CPM was employed to understand the clathrin-dependant cellular internalization ${ }^{63}$. Dynamin (GTPase) dependency in endocytosis of cells was probed by using dynasore ${ }^{64}$. Additionally, nystatin, which is a sterolbinding agent, was used to study clathrin-independent inhibition of endocytosis. Nystatin is known cause dissembly of caveolae and cholesterol in the membrane ${ }^{65,66}$. We repeated our ICP analysis of internalized gold with these four inhibitors of various major pathways of cellular internalization. If the addition of any of these internalization inhibitors were to reduce the percent of treated gold found in the cellular pellet, we would interpret this as a pathway which our endocytosis was routed. From this study, however, we found that none of these inhibitory treatments were able to reduce the internalization of the gold (Supplementary Fig. 6). We found the lack of impact on internalization by these inhibitors perplexing, as these pathways were involved in majority of the cellular internalization and approximately half of the gold was being internalized. A microarray analysis was then used to identify changes in gene expression for pathways related endocytosis (Supplementary Fig. 7). Through this pathway map, we observed no change in the gene expression for clarthrindependent endocytosis (ARF6, PIPK1A, PLD1), clathrinindependent endocytosis (ARF6, SRC, PIP5K1A, HRAS, PLD1), or lipid raft formation (PIP2, MHC1, PIP3). This may indicate internalization through non-classical means or through mechanical forces generated as gold is reduced along the cell membrane forming bonds with cellular proteins. However, there are changes in gene expression for processes within this mapping. Within this map, genes those are the most heavily downregulated are associated with cation binding (TGFBR2, ERBB4, CBLB, GIT2), and those upregulated are associated with cell stress and protein refolding (HSPA1A). However, the participation of immunoglobulin heavy constant alpha 1 (IGHA1) protein during the bio-reduction of Au-PEG clusters to form AuNPs (Table 1) supports the receptor-mediated phagocytosis as one of plausible pathways of cellular internalization for these clusters.

In vivo theranostics. From our application of ionic gold clustered with PEG, we have developed a unique system wherein gold nanoparticles are reduced by cellular biomolecules those are able to retain their functionality, including the capacity to guide the remaining cluster to the nucleus. The intracellular formation and nuclear migration of these gold nanoparticles presents a high potential for theranostic photothermal ablative application. To discover the theranostic potential of the intracellular generation of functional gold nanoparticles, we applied our ionic Au-PEG clusters to MCF-7 xenografts in J:NU mice compared with control injections of ionic Na-PEG (Supplementary Fig. 8). Intratumoral injections of ionic Au-PEG clusters presented fluorescence contrast via IVIS imaging with an excitation wavelength of 430 $\mathrm{nm}$ and an emission wavelength of $840 \mathrm{~nm}$. Although some fluorescence signal at $430 \mathrm{~nm}$ excitation and $840 \mathrm{~nm}$ emission was observed, the lack of strong fluorescence signal in vitro was presumably due to the differences in microenvironment and cell density which could result in variation of sizes of nanoparticles as well as in concentration. This observed fluorescence emission is not uncommon for ultrasmall gold nanoclusters $(<5 \mathrm{~nm})$, wherein the emission wavelength can be tuned from 385 to $866 \mathrm{~nm}^{67}$. From our system, wherein gold nanoparticles were generated intratumorally, fluorescence contrast was observable above background as early as one day after injection of ionic Au-PEG clusters (Fig. 7a-c). We observed increasing contrast between injection sites and off-site locations for up to 2 days, which would then dissipate after one week (Fig. 7a). The increase in fluorescence contrast between the injection site and off-site locations over this 2-day period suggests that nanoparticles may be continually forming at the injection site for up to 2 days beyond the injection time point. This fluorescence contrast between injection site and off-site (no treatment) was not observed for ionic Na-PEG injections at all the time points. To investigate therapeutic potential, tumors injected with either Au-PEG or Na-PEG would receive laser treatments using $500 \mathrm{~mW} 633 \mathrm{~nm}$ continuous wave laser for $3 \mathrm{~min}$ (+laser) or $0 \mathrm{~min}$ (-laser) periods with an iPad-mounted IR camera to monitor and quantify the temperature changes. When applications of laser ablation were applied, tumor temperatures would rise to $51.2 \pm 1.7^{\circ} \mathrm{C}$ for successful intratumoral Au-PEG treatments and $40.8 \pm 1.0^{\circ} \mathrm{C}$ for $\mathrm{Na}-\mathrm{PEG}$ treatments (Fig. 7b). Successful hyperthermia is commonly defined as heating tissue at $>45^{\circ} \mathrm{C}$ for several minutes (5-10 $\mathrm{min})^{68,69}$. Lesions/scabbing and tumor remediation occurred for tumors with successful applications of Au-PEG with two or fewer laser applications in all but one mouse $(n=5)$. Laser applications made to successful Au-PEG treatments would reduce the apparent fluorescence contrast when compared against $\mathrm{Au}-\mathrm{PEG}$ treatments without laser treatment (Fig. 7a). This apparent reduction in fluorescence contrast from laser treatment is likely due to the resultant hyperthermic process, either through loosening cell membranes and destroying tissue 
a
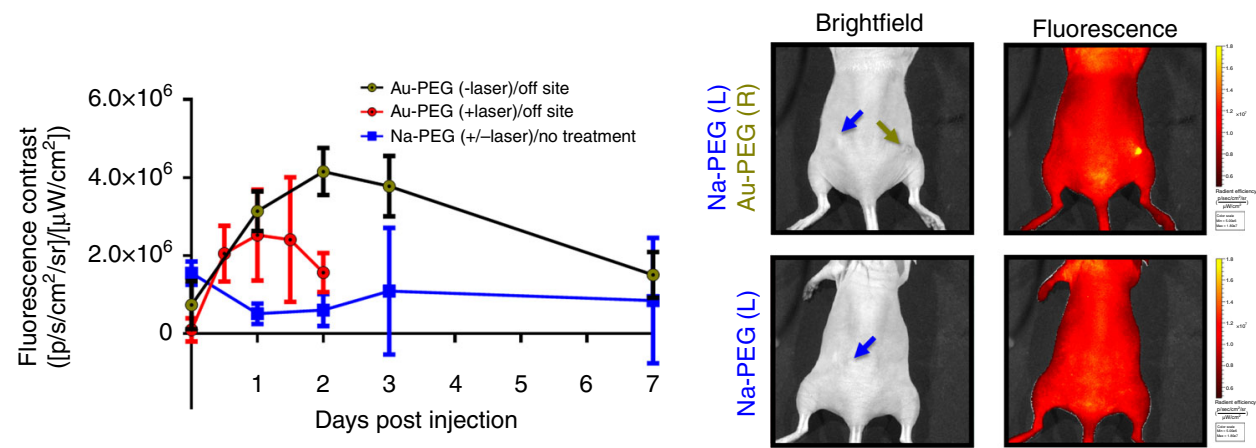

b

Max temperature

$\left({ }^{\circ} \mathrm{C}\right)$
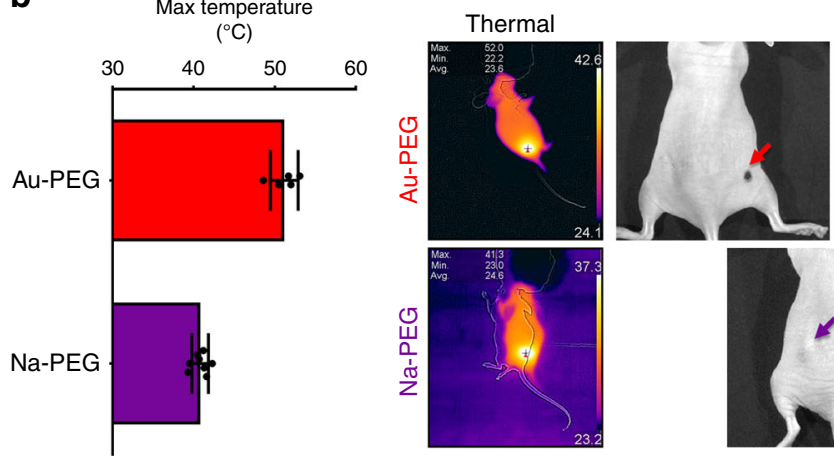

Brightfield

C
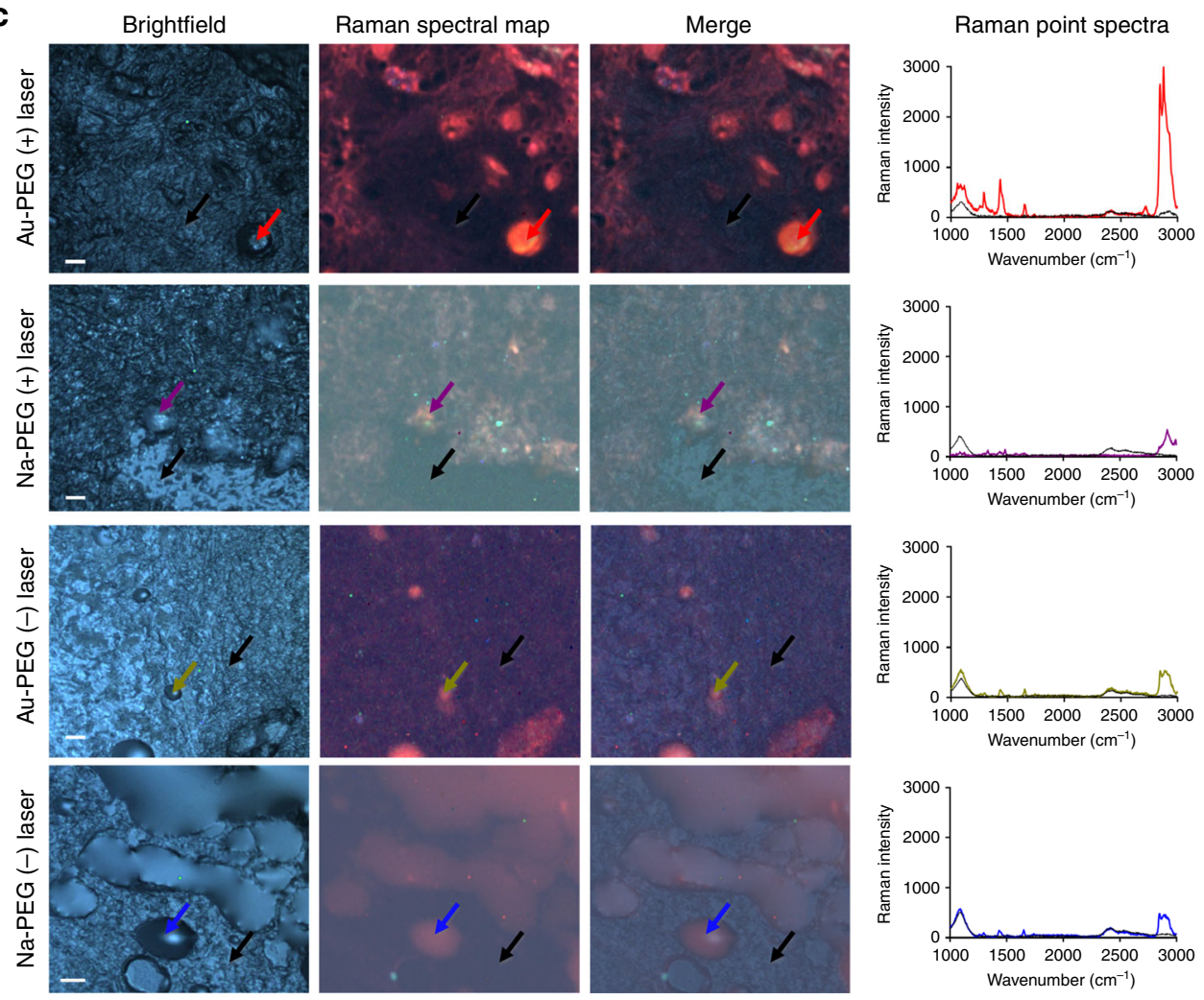

Fig. 7 In vivo efficacy of the gold nanoparticles generated through vectorized biomineralization of ionic gold. a Fluorescence contrast afforded via in vivo imaging system (IVIS)-based imaging $\left(43 \mathrm{E}_{\mathrm{Ex}} 84 \mathrm{E}_{\mathrm{Em}}\right.$ ) between on-site and off-site across one week period for treatments of Au-PEG with no photothermal treatment (yellow), Au-PEG with photothermal treatment (red), or Na-PEG (blue) with bright field and fluorescence images representative of Au-PEG or Na-PEG-treated mice (three days post injection) with arrows indicating intratumoral injection sites for Na-PEG (blue) and Au-PEG (yellow). Error bars are standard deviation of the mean ( $n \geq$ 3 biologically independent animals). b Photothermal effect of intratumorally generated gold nanoparticles via 3 min applications of $500 \mathrm{~mW} 633 \mathrm{~nm}$ laser as represented by maximum surface temperature from the tumors either treated with Na-PEG (purple) or Au-PEG (red) with thermal and bright field images representative of Na-PEG (purple) or Au-PEG (red)-treated mice after two laser applications (thresholding contrast for thermal images are automatically read via the iPad mounted FLIR software). Error bars are standard deviation of the mean ( $n \geq 5$ laser treatments). c Bright field, Raman spectral mapped and merged images of excised tumors from mice treated with Au-PEG ( \pm laser ablation) or Na-PEG ( \pm laser ablation) with corresponding Raman point spectra for positions designated with arrows. Scale bars are 20 um. 
(allowing nanoparticle escape) and/or through the addition of thermal energy modifying the reaction kinetics (forming nonfluorescent nanoparticles). Hyperthermia causes cell damage by loosening cell membranes and denaturing proteins ${ }^{70,71}$, and hyperthermic laser applications have been demonstrated previously to spontaneously reduce nanoparticles through laser heating ${ }^{72-74}$. It is likely that a combination of these effects are taking place, wherein photothermal applications are simultaneously loosening tissue for the escape of fluorescent gold nanoparticles and unreacted ionic gold as well as initiating laserinduced gold nanoparticle formation (Supplementary Fig. 9). Applications of Au-PEG which were unsuccessfully applied intratumorally, due to the small size of the tumors (resulting in an intradermal application), would result in diffusive fluorescence with contrast between tumor and off-site no greater than Na-PEG (Supplementary Fig. 10). When ablative laser applications were applied to intradermal injections of Au-PEG (opposed to intratumoral injections), tumor temperatures would rise to $40.7 \pm$ $2.3^{\circ} \mathrm{C}$ (no significant difference than Na-PEG). The low fluorescence contrast and lack of photothermal effect from intradermal injections of Au-PEG (compared to intratumoral injections) provided us with near immediate feedback and utility of intratumorally generated gold nanoparticles towards theranostic application. This is because, after failed intratumoral injections, we were able to acquire fluorescent images for confirmation of the success of our application. This allowed us to predict if photothermal laser application would provide therapeutic effect or if we should rather wait for fluorescence to reduce background (by waiting one week) before attempting a second injection attempt. Therefore, if we applied laser therapy to unsuccessful injections, we would observe no hyperthermia impact (on-site or off-site). To further investigate the ionic AuPEG cluster for theranostic applications focusing on the intratumoral generation of gold nanoparticles, organs (tumors, sentinel lymph nodes, liver, kidneys, and spleen) were harvested and imaged with either Raman mapping (Fig. 7c, Supplementary Fig. 11) or stained by $\mathrm{H} \& \mathrm{E}$ for bright field imaging (Supplementary Fig. 12). From Raman mapping, we found increased Raman signal indicating plasmonic enhancement in tumors treated with Au-PEG which had received photothermal ablation compared with the other treatments (Fig. 7c). This plasmonic enhancement, found only in the section of the tumor treated with $\mathrm{Au}-\mathrm{PEG}$ clusters and laser therapy, indicates that photothermal ablation may induce or modify nanoparticle formation in a manner separate from the cellular reduction. The application of ionic Au-PEG clusters to generate fluorescent and photothermally active nanoparticles intratumorally resulted in no observable pathological effects through our short-term experiment regardless of the success of the administration. Additionally, we demonstrated that hyperthermic laser applications could be applied to modify the nanoparticle formation intratumorally.

\section{Discussion}

In summary, we have demonstrated a simplistic nano-scaled delivery system that combines ionic gold and PEG, which enables the progressive reduction and formation of plasmonic gold nanoparticles. These nanoparticles are both fluorescent and provide a route for ablative therapies through photothermal heating. We have characterized this process through the identification of associated proteins combined with their entailing molecular processes and subcellular locations. We have determined that our process does not destroy the integrity of its associated proteins and requires considerably lower concentrations of ionic gold and time as compared to the previous literature methods. For this nanotheranostic platform, we understand that a cellular-driven synthesis may be perceived as a major drawback as it poses as an element outside of control. However, this lack in control may pose as a potential benefit wherein theranostic nanoparticles are generated differentially, dependent on the cellular machinery and microenvironment. Further to this, we envisage to observe this effect in normal control cells in comparison to the cancer cells and develop a more optimized generation of biomineralized plasmonic nanoparticles which can efficiently be used for hyperthermia applications in vivo. We envision future applications similar to this presented work, wherein the treatment focuses on optimization of the nanostructures to propel the next major advancement in nanomedicine. These envisioned fluid-form treatments will utilize and rely on cellular machinery dependent on the local pathology of the tissue providing highly specific opportunities for sensing, therapy, or control over local physiology.

\section{Methods}

Materials. Chloroauric acid (SKU: 254169-5G), bi-hydroxyl terminated polyethylene glycol $(\mathrm{Mn}=10,000 \mathrm{~g} / \mathrm{mol})$ (SKU: 8218811000$)$, sodium chloride (SKU: 746398-500G), $\beta$ ME (SKU: M6250-100ML), dimethyl sulfoxide (SKU: 276855100ML), fetal bovine serum (SKU: TMS-013-B), thiazolyl blue tetrazolium bromide (SKU: M5655-500MG), phosphate buffered saline (SKU: P3813-10PAK), Dulbecco's modified Eagle medium (SKU: D7777-10X1L), trypsin-EDTA (SKU: T4049$500 \mathrm{ML}$ ), aluminum potassium sulfate dodecahydrate (SKU: A6435-100G), and sodium iodate (SKU: 71702-25G) were purchased from Sigma-Aldrich unless otherwise stated. All components for SDS-PAGE and protein extraction kit were purchased from BioRad Laboratories. Histology grade ethanol was purchased from Thermo Scientific. Histology grade xylene was purchased from VWR. Eosin Y Hematoxylin hydrate, and Phloxine B were purchased from Acros Organics.

Au-PEG cluster synthesis. In a $20 \mathrm{ml}$ scintillation vial, 0.015 mmoles of $\mathrm{HAuCl}$ and $500 \mathrm{mg}$ of PEG $(\mathrm{Mn}=10,000 \mathrm{~g} / \mathrm{mol})$ were mixed with $2 \mathrm{ml}$ of carbon-filtered deionized water $(0.2 \mu \mathrm{m}$ cellulosic membrane, $\mathrm{pH} 4.0)$. This mixture was incubated in a $37^{\circ} \mathrm{C}$ water bath for $30 \mathrm{~min}$ before being diluted for further use.

Na-PEG cluster synthesis. In a $20 \mathrm{ml}$ scintillation vial, 0.015 mmoles of $\mathrm{NaCl}$ and $500 \mathrm{mg}$ of PEG $(\mathrm{Mn}=10,000 \mathrm{~g} / \mathrm{mol})$ are mixed with $2 \mathrm{ml}$ of carbon-filtered deionized water $(0.2 \mu \mathrm{m}$ cellulosic membrane, $\mathrm{pH} 4.0)$. This mixture was incubated in a $37^{\circ} \mathrm{C}$ water bath for $30 \mathrm{~min}$ before being diluted 100 fold with carbon-filtered deionized water $(0.2 \mu \mathrm{m}$ cellulosic membrane, $\mathrm{pH}=7)$.

TEM measurements. For TEM, Au-PEG clusters were diluted 100-fold with carbon-filtered deionized water $(0.2 \mu \mathrm{m}$ cellulosic membrane, $\mathrm{pH}=7)$ and $2.5 \mu \mathrm{l}$ of the diluted Au-PEG clusters were placed on a 300-mesh carbon film supported by a copper grid and allowed to stabilize for $2 \mathrm{~min}$. A filter paper was then used to remove liquid for thin film formation and then allowed to air dry while covered. Images were obtained using a Jeol 2010 cryo-electron microscope operating at $200 \mathrm{kV}$, and using different degrees of defocus to obtain an adequate bright contrast. Images were recorded on a Gatan UltraScan $2 \mathrm{k} \times 2 \mathrm{k}$ CCD. These CCD images were processed and analyzed with ImageJ (http://rsbweb.nih.gov/ij/) version 1.48.

Scanning electron microscopy and electron dispersion spectroscopy. For SEMEDS, Au-PEG clusters were diluted 100-fold with carbon-filtered deionized water $(0.2 \mu \mathrm{m}$ cellulosic membrane, $\mathrm{pH}=7)$ and several $3 \mu \mathrm{l}$ drops of the diluted Au-PEG clusters were placed on a strip of copper tape placed on an SEM sample grid and allowed to stabilize for $5 \mathrm{~min}$. A filter paper was used to remove liquid for thin film formation and then allowed to air dry while covered. Images were obtained using a Hitachi S4700 scanning electron microscope with Oxford Instruments ISIS EDS Xray Microanalysis System. SEM images were captured using a $10 \mathrm{kV}$ accelerating voltage, a $10 \mu \mathrm{A}$ emission current, and a $12 \mathrm{~mm}$ working distance, adjusting sample height for coarse focus and low degrees of defocus to obtain secondary electron images and EDS. Images were recorded using a Centaurus BSE detector.

UV-Vis absorption studies. For collecting the UV-Vis spectra, $1 \mathrm{ml}$ of the as prepared Au-PEG clusters were used. For calibration, the blank consisted of carbon-filtered deionized water $(0.2 \mu \mathrm{m}$ cellulosic membrane, $\mathrm{pH}=7)$. The absorption spectra were acquired in scanning mode for the range of $200-1000 \mathrm{~nm}$ using a GeneSys $10 \mathrm{~S}$ UV-Vis spectrophotometer (Thermo Scientific, Rockford, IL). Measurements were taken at every $0.1 \mathrm{~nm}$ interval.

Mammallian cell culture. MCF-7 cells (ER $(+)$ human breast cancer cells) sourced from ATCC (catalog no. HTB-22) were cultured in Dulbecco's modified Eagle's medium (DMEM; Sigma) supplemented with $10 \%$ fetal bovine serum (FBS) and $1 \mathrm{x}$ 
Penstrep in T25 culture flasks (Cellstar, Germany) and were incubated at $37^{\circ} \mathrm{C}$ in a $99 \%$ humidified atmosphere containing $5 \% \mathrm{CO}_{2}$. Cells were regularly passaged by trypsinization with $0.1 \%$ trypsin (EDTA $0.02 \%$, dextrose $0.05 \%$, and trypsin $0.1 \%$ ) in DPBS (pH 7.4). Non-synchronized cells were used for all the experiments.

Cell growth inhibition studies with MTT assay. MCF-7 cells were seeded at 10,000 cells/well in DMEM with $10 \%$ FBS and 1x Penstrep (200 $\mu \mathrm{l} /$ well) in a 96well plate and incubated at $37^{\circ} \mathrm{C}$ in a $99 \%$ humidified atmosphere containing $5 \%$ $\mathrm{CO}_{2}$ for $24 \mathrm{~h}$. Cell treatments were as follows: control, Na-PEG, nifuroxazide-PEG, $\mathrm{Au}-\mathrm{PEG}$, and $\mathrm{Au}^{3+}$. Each treatment of the cells had concentrations $0.05-0.6 \mathrm{mM}$. After treatment, cells were incubated for $44 \mathrm{~h}$. After $44 \mathrm{~h}, 20 \mu \mathrm{l}$ MTT solution ( $5 \mathrm{mg} / \mathrm{ml}$ Thiazolyl blue tetrazolium bromide) was added to each well and the plate was incubated for an additional $4 \mathrm{~h}$. Media was then aspirated from each well and $200 \mu \mathrm{l}$ dimethyl sulfoxide (DMSO) was added. The plate absorbance was then read at $570 \mathrm{~nm}$ wavelength using Gen5 Microplate Reader and Imager Software. For investigating the reaction with ionic gold and Thiazolyl blue tetrazolium bromide, identical concentrations were used, without cells.

\section{Cell culture treatment with Au-PEG clusters for transmission electron} microscopy. Samples of Au-PEG were diluted in full cell media to $0.24 \mathrm{mM} \mathrm{Au}^{3+}$ by mixing $0.5 \mathrm{ml} \mathrm{Au}$-PEG clusters with $15 \mathrm{ml}$ cell media. After removing old media, this mixture was incubated on a monolayer of MCF7 cells (grown in T-25 flasks until $\sim 80 \%$ confluency) growing at $37^{\circ} \mathrm{C}$ with $5 \% \mathrm{CO}_{2}$ for $4 \mathrm{~h}$. After this incubation period, growth medium was discarded and cell monolayer was washed with DPBS before trypsinizing the treated cells. Cells were harvested in small $1.5 \mathrm{ml}$ centrifuge tubes and collected in DPBS before fixing. After the cell pellet was fixed in a Karnovsky's fixative in phosphate buffered 2\% glutaraldehyde and 2.5\% paraformaldehyde. Microwave fixation was used with this primary fixative, and the tissue is then washed in Sorenson's phosphate buffer with no further additives. Microwave fixation was also used with the secondary $2 \%$ osmium tetroxide fixative, followed by the addition of $3 \%$ potassium ferricyanide for $30 \mathrm{~min}$. After washing with water, saturated uranyl acetate was added for enbloc staining. The tissue was dehydrated in a series of increasing concentrations of ethanol. Acetonitrile was used as the transition fluid between ethanol and the epoxy. Infiltration series was done with an epoxy mixture using the epon substitute Lx112. The resulting blocks were polymerized at $90^{\circ} \mathrm{C}$ overnight, trimmed and ultrathin sectioned with diamond knives. Sections were stained with uranyl acetate (2\%) and lead citrate (3\%) and examined or photographed with a Hitachi H600 transmission electron microscope at $75 \mathrm{kV}$.

Enhanced darkfield hyperspectral imaging. Optical and hyperspectral images were captured at $\times 60$ magnification under enhanced darkfield illumination using the CytoViva hyperspectral imaging system (Auburn, AL). This hyperspectral imaging system couples an enhanced dark-field illuminator with optical and hyperspectral CCD cameras. The hyperspectral images, also called datacubes, were collected using the "push broom" method. The spectral data $(400-1000 \mathrm{~nm})$ was acquired 1 pixel row at a time. The acquisition was facilitated by a motorized stage. The hyperspectral analysis software ENVI compiled this spectral and spatial data into a datacube, in which each pixel contained spectral data.

Spectral libraries corresponding to the reduced gold nanoparticles were built from the images of exposed MCF-7 cells. These libraries were filtered against a negative control image (cells only, Supplementary Fig. S1e) to ensure no falsepositive mapping of the nanoparticles. Using the spectral angle mapper (SAM) algorithm, the spectral libraries were compared to their respective images.

Control treatments with Au-PEG clusters. Both $150 \mu \mathrm{l}$ of as-prepared Au-PEG clusters and $250 \mu \mathrm{l}$ of media either fresh or having been used to incubate cells for $\sim 24 \mathrm{~h}$ ('spent' media) were mixed in a $2 \mathrm{ml}$ microcentrifuge tube and incubated in a water bath for $24 \mathrm{~h}$. From these samples, $2.5 \mu \mathrm{l}$ was collected for TEM analysis as described in the "TEM measurements" section.

$2 \mathrm{ml}$ of as-prepared Au-PEG clusters were added to an empty poly-L-lysinetreated culture plate. This container was incubated at $37^{\circ} \mathrm{C}$ in a $99 \%$ humidified atmosphere containing $5 \% \mathrm{CO}_{2}$ for $24 \mathrm{~h}$. From these samples, $2.5 \mu \mathrm{l}$ would be extracted for TEM analysis as described in the TEM measurements section.

\section{Cell culture treatment with Au-PEG clusters for Raman spectroscopic ana-}

Iysis. Samples of Au-PEG were diluted in full cell media to $0.24 \mathrm{mM} \mathrm{Au}^{3+}$ by mixing $0.5 \mathrm{ml} \mathrm{Au}$-PEG clusters with $15 \mathrm{ml}$ cell media. After removing old media, this mixture would incubate on a monolayer of MCF7 cells (grown on glass slides until $~ 80 \%$ confluency) growing at $37^{\circ} \mathrm{C}$ with $5 \% \mathrm{CO}_{2}$ for $4 \mathrm{~h}$. After this incubation period, growth medium was discarded and cell monolayer was washed with DPBS before fixing with $37 \%$ formaldehyde solution. Raman spectra were acquired on cells incubated with Au-PEG treatment and on control cells lacking treatment in reflection mode (LabRAM HR 3D Horiba confocal microscope). Laser light was focused through a $\times 100$, NA 0.8 objective into the sample plane and the scattering was collected in the reflection geometry using the spectrograph coupled with an Andor Newton back-illuminated EMCCD camera. The excitation wavelength for the measurements was set to $633 \mathrm{~nm}$, and the power was set to $8 \mathrm{~mW}$ at the sample with a $0.2 \mathrm{~s}$ acquisition time. Raman shift from 1000 to $3050 \mathrm{~cm}^{-1}$ was collected at
$8 \mathrm{~cm}^{-1}$ spectral resolution. Intensities of select vibrational modes were selected to generate the Raman images.

Cell fractionation of treated cells. Samples of Au-PEG were diluted in full cell media to $0.24 \mathrm{mM} \mathrm{Au}^{3+}$ by mixing $0.5 \mathrm{ml}$ Au-PEG clusters with $15 \mathrm{ml}$ cell media. After removing old media, this mixture was administered on a monolayer of MCF7 cells (grown in T-25 flask $\sim 80 \%$ confluency) growing at $37^{\circ} \mathrm{C}$ with $5 \% \mathrm{CO}_{2}$ for $4 \mathrm{~h}$ The cells were then trypsinized and centrifuged at $20,000 \times g$ to form a cell pellet. The trypsin was removed, and the pellet suspended in $1.5 \mathrm{ml}$ DPBS before tip sonication ( $5 \mathrm{~s} \mathrm{ON}, 2 \mathrm{~s}$ OFF at $1 \mathrm{~A}$ for $2 \mathrm{~min}$ ) was used to rupture the cells. Ruptured cells were separated into cell fractions ${ }^{1}$ and homogenate was first centrifuged at $600 \times g$ to pellet unbroken cells. Supernatant was collected and centrifuged at $15,000 \times g$ for $5 \mathrm{~min}$. The pellet was separated and the supernatant was collected and centrifuged at $100,000 \times g$ for $60 \mathrm{~min}$. The pellet was separated and the supernatant was collected and centrifuged at $300,000 \times g$ for $120 \mathrm{~min}$.

Using an Eppendorf (Hamburg, Germany) 5424R microcentrifuge for centrifugation speeds lower than $100,000 \times g$, and a Beckman Coulter (Indianapolis, IN) Optima MAX-XP Ultracentrifuge for speeds above 100,000 $\times g$. Pelleted fractions were suspended in $1 \mathrm{ml}$ DPBS (pH 7.4) before SDS-PAGE or Raman analysis. SDS-PAGE and Raman samples were prepared immediately after fractionization.

Raman spectroscopic analysis. Raman measurements were taken using a Nanophoton Raman instrument at Frederick Seitz Materials Research Laboratory Central research facilities, UIUC ( $532 \mathrm{~nm}$ laser). For each spectrum, a grating $\left(600 \mathrm{~mm}^{-1}\right)$ scan was taken over the range of $1000-3000 \mathrm{~cm}^{-1}$ at $0.2 \%$ laser power for 1 min (with $\times 20$ objective). An average of 20 spectra was recorded per sample. Spectra were exported as.txt files and plotted using Graphpad prism.

SDS-PAGE analysis of cell fractions. A BioRad Mini-PROTEAN ${ }^{\oplus}$ tetra vertical electrophoresis cell was loaded with $2 \times 5-20 \%$ gradient Mini-PROTEAN gels and the electrophoresis cell filled to the appropriate fill line with $1 \mathrm{x}$ Tris/Glycine SDS buffer. Two portions of nuclear protein fractions from cells treated with Au-PEG $\mathrm{Na}$-PEG, or untreated were admixed with $4 \mathrm{x}$ Laemmli sample buffer (BioRad) either containing $2.5 \% \beta \mathrm{ME}$ or with $1 \mathrm{x}$ Tris/glycine SDS buffer in the same volume with pipetting to addmix. From these prepared protein samples $20 \mu \mathrm{l}$ was added to wells $2-4$ and $6-8$, with $5 \mu \mathrm{l}$ of Precision Plus $(10-250 \mathrm{kDa})$ protein ladder in lanes 5 and 9 . After allowing the samples to settle in their respective wells for 5 min the cover of the electrophoresis cell was added, power turned on to $200 \mathrm{~V}$ and allowed to run $\sim 35 \mathrm{~min}$ (the dye front $<1 \mathrm{~cm}$ from the edge of the gel). For LC-MS, the nuclear fraction extracted from cells treated with Au-PEG were allowed to run for $>3 \mathrm{~h}$ (until all of the ladder proteins had run off the gel) without protein from other cell treatments.

Coomassie Blue total protein stain. Gel was briefly rinsed with $\mathrm{DI} \mathrm{H}_{2} \mathrm{O}$ before being immersed in protein fixing solution (10\% acetic acid, $10 \%$ methanol, in $\mathrm{Di}$ $\mathrm{H}_{2} \mathrm{O}$ ) for $30 \mathrm{~min}$ with gentle rocking. After fixing for $30 \mathrm{~min}$, the gel was stained with $1 \mathrm{x}$ Coomassie Blue staining solution (BioRad) for $2 \mathrm{~h}$ with gentle rocking. After staining, the gel was submerged in destaining solution (10\% acetic acid in DI $\mathrm{H}_{2} \mathrm{O}$ ) for $24 \mathrm{~h}$, replacing the destaining solution as it became saturated with residual Coomassie stain. After the gel was adequately destained, a BioRad gel-dock imaging system was used to image the stained gel. The images were contrast enhanced, and band intensities analyzed with ImageJ. The uncropped images for these coomassie-stained SDS-PAGE gels have been shown in Supplementary Fig. 3.

Protein extraction via passive elution and LC-MS analysis. In order to separate proteins, bound to gold nanoparticles from unbound, we used the nuclear fractions from MCF-7 cells treated with Au-PEG and allowed it to run through gel electrophoresis in excess of time $(>3 \mathrm{~h})$. The top portion of the gel containing the wells of the gel (the top $1.5 \mathrm{~cm}$ ) were excised from the bulk of the gel with a scalpel and placed into separate $15 \mathrm{ml}$ centrifuge tubes. The gel pieces were ground inside their respective containers with sterile cleaned metal weighing spatulas before mixing with minimal elution buffer $(50 \mathrm{mM}$ Tris- $\mathrm{HCl}, 150 \mathrm{mM} \mathrm{NaCl}$, and $0.1 \mathrm{mM}$ EDTA at $\mathrm{pH}$ 7.5), but ensuring that the gel pieces were completely immersed. These immersed gel fragments containing protein were incubated at room temperature on a rocker for $48 \mathrm{~h}$. After incubation, samples were centrifuged at $5000 \times g$ for $10 \mathrm{~min}$ before removing the supernatant for LC-MS analysis via partial trypsinization digestion performed in the Roy J. Carver Biotechnology Center (CBC).

Inhibitor study. A T-175 flask of MCF7 cells at $80 \%$ confluency was split and plated (at equal cell density) into $15 \mathrm{~T}-25$ culture flasks. Cells were grown for $24 \mathrm{~h}$ before being incubated with various endocytic inhibitors. Inhibitor formulations were made with reconstituted medium containing $\mathrm{NaN}_{3}$ and deoxyglucose, $\mathrm{CPM}$ nystatin and dynasore at a concentration of $10,50,28 \mu \mathrm{M}, 180 \mathrm{nM}$ and $80 \mu \mathrm{M}$, respectively ${ }^{2-4}$. Cells were incubated with inhibitors for $1 \mathrm{~h}$ at ambient condition. Inhibitors were then replaced with Au-PEG diluted in full cell media to $0.24 \mathrm{mM}$ $\mathrm{Au}^{3+}$ by mixing $0.5 \mathrm{ml}$ Au-PEG clusters with $15 \mathrm{ml}$ cell media. All the treatments were performed in triplicate. Cells treated with ionic Au-PEG clusters in absence of 
inhibitors were used as control. After the required incubation time of $4 \mathrm{~h}$, cell pellets and supernatants were collected for each sample and subjected to further ICP-MS analysis to determine the intracellular amount of gold.

Flow cytometry analysis. To further characterize the cytotoxicity of our ionic gold delivery platform, we performed flow cytometry analysis. Cells (MCF-7, 10,000 cells/well) were grown in 24-well plate for $24 \mathrm{~h}$ before treating with different samples prepared for this study for $4 \mathrm{~h}$. At the end of incubation, cells were trypsinized and collected in $0.2 \%$ FBS containing DPBS. Samples were analyzed using a Guava EasyCyte Plus Flow cytometer. For each sample, data from 5000 single cell events were collected for $3 \mathrm{~min}$, in triplicates, and forward scatter vs. side scatter information were recorded. The results were analyzed and plotted using Flowpy.

Animal studies. To investigate potential theranostic applications pre-clinically, we generated and treated MCF-7 xenograft tumors in female J/NU immunodeficient double knockout mice. The experimental protocol received ethical approval and was approved by the Institutional Animal Care and Use Committee (IACUC), University of Illinois at Urbana-Champaign, and satisfied all University and National Institutes of Health (NIH) rules for the humane use of laboratory animals. All the animal experiments were complied by the relevant ethical regulations for animal testing and research and carried out in accordance with the approved guidelines. Female J/Nu mice (2-3 weeks old) were bought from Jackson Laboratories, USA. Upon arrival, mice were allowed 2 weeks for acclimation. Animals were group housed with free access to food and water, and individuality marked through ear punching. We injected $10^{6}$ cells in matrigel on the hind flanks of $n=12$ mice with 1 injection per side. Of these injections, 16 tumors grew to usable size $\left(\sim 5 \times 5 \mathrm{~mm}^{2}\right)$. There were 5 mice with 2 tumors, 6 with only 1 , and 1 mouse which did not have any tumors of usable size. These 16 tumors received intratumoral injections of either Na-PEG or Au-PEG. Mice which had tumor growth to suitable size $\left(\sim 5 \times 5 \mathrm{~mm}^{2}\right)$ on both flanks received two different treatments to both tumors (i.e. Au-PEG or Na-PEG and tlaser ablation). Tumors injected with either Au-PEG or Na-PEG received photothermal ablative therapy using $500 \mathrm{~mW} 633 \mathrm{~nm}$ continuous wave laser for $5 \mathrm{~min}$ (+laser) or $0 \mathrm{~min}$ (-laser) periods with IR camera (FLIR technologies) to monitor and quantify the temperature changes. From these groups, we had four tumors with Na-PEG injections and $0 \mathrm{~min}$ of laser treatment; three tumors with $\mathrm{Na}-\mathrm{PEG}$ injections and $5 \mathrm{~min}$ of laser treatment; four tumors with Au-PEG injections and 0 min of laser treatment; five tumors with Au-PEG injections and 5 min of laser treatment. For all tumors which were successfully ablated, treatments were no longer made for those mice. Injections of $40 \mu \mathrm{l}$ were administered using 32 gauge syringe needles over $2 \mathrm{~min}$ under isoflurane anesthesia $(2.5 \%$ in oxygen) with an oxygen flow rate of $11 / \mathrm{h}$.

IVIS imaging studies. Animals were serially imaged at 1,24, 48, 72, and $168 \mathrm{~h}$ after treatment at $430 \mathrm{~nm}$ excitation and $840 \mathrm{~nm}$ emission wavelengths. Animals which received photothermal ablative intervention were imaged at these same time points and additionally $30 \mathrm{~min}$ after laser treatment (1.5, and 24.5 , and $48.5 \mathrm{~h}$ ). During imaging, animals were anesthetized with isoflurane (2.5\% in oxygen) with an oxygen flow rate of $1 \mathrm{l} / \mathrm{hr}$.

Photothermal ablative therapy with IR-Imaging. Photothermal ablative therapy was applied using $500 \mathrm{~mW} 633 \mathrm{~nm}$ continuous wave laser (RLTMRL-635-500-5, Roithner Lasertechnik) with $40 \mathrm{~mm}^{2}$ beam diameter for $5 \mathrm{~min}$ (+laser) or $0 \mathrm{~min}$ (-laser) periods and monitored with IR camera (FLIR technologies) to visualize and quantify the temperature changes. Anesthesized mice were placed on the laser stand mount, using a machine-etched crosshair, so that the beam path would intersect directly with the tumor. Upon completion of the laser therapy, a neutral density filter with $100 \%$ OD was placed in front of the beam, blocking the path of the laser. See Supplementary Fig. S9 for laser stand setup. An iPad-mounted IRcamera (FLIR Technologies) was used to obtain snapshot thermal images at $2.5 \mathrm{~min}$ after photothermal applications with a laser. Temperature maxima for these images were considered as photothermal treatments representative of surface temperature. During photothermal treatments and IR-imaging animals were anesthetized with isoflurane ( $2.5 \%$ in oxygen) with an oxygen flow rate of $11 / \mathrm{h}$.

Histology sectioning. At the end of the experiment, animals were sacrificed via anesthesia overdose. Organ tissues (tumors, sentinel lymph nodes, liver, kidneys, and spleen) were immediately excised and frozen in cassettes containing optimal cutting temperature (OCT) compound. Embedded tissue blocks were clamped in microtome and sectioned at $6 \mu \mathrm{m}$ thickness. Tumor and sentinel lymph node slices were either left unstained (for Raman mapping) or stained with hematoxylin and eosin (H\&E) for bright field imaging.

H\&E staining. Slides were first fixed for $5 \mathrm{~min}$ in $4 \%$ PFA. Immediately following fixation, slides were rinsed in cold PBS. Slides were then stained with H\&E using standard protocol. Briefly, slides were immersed for $8 \mathrm{~min}$ in Mayer hematoxylin solution, followed by a $2 \mathrm{~min}$ wash in warm, slow running water. Then, slides were blued with two dips in lithium carbonate. Slides were again washed in warm slow running water. Slides were then immersed in $80 \%$ ethanol for $2 \mathrm{~min}$, followed by $5 \mathrm{~min}$ in eosin. Slides were dehydrated by performing sequential immersions in ethanol, $4 \times 2 \mathrm{~min}$ in $95 \%$ ethanol (in two separate containers), $4 \times 2 \mathrm{~min}$ in $100 \%$ ethanol (in two separate containers), and finally $3 \times 3 \mathrm{~min}$ immersions in xylene (in three separate containers). Coverslips were then applied using xylene-based Permount.

Raman-mapped histology. Unstained tissue sections were imaged in the reflection mode via LabRAM HR 3D Horiba confocal microscope with $532 \mathrm{~nm}$ wavelength excitation laser with power set at $8 \mathrm{~mW}$. The laser light was focused through a LWD Visible $\times 20$, NA 0.40 , objective into the sample plane and scattering was recorded in the reflection geometry using a spectrograph coupled with an Andor Newton back-illuminated EMCCD camera. An acquisition time of $1 \mathrm{~s}$ was used. The Raman shift from 310 to $3410 \mathrm{~cm}^{-1}$ was recorded with a spectral resolution of $3 \mathrm{~cm}^{-1}$.

Reporting summary. Further information on research design is available in the Nature Research Reporting Summary linked to this article.

\section{Data availability}

The authors declare that the data supporting all the findings of this study are available within the article and Supporting Information files. Zip files for Microarray data can be downloaded using the following link (https://uofi.box.com/s/

b10c77c0anr5q4v7sm9k313w5r8f $92 \mathrm{z})$; CSV files for protein MS data can be downloaded using the following links: Top gel portion (https://uofi.box.com/s/ 916j732etrh2uqy17o0f3p8aqvp5p15a); Bottom gel portion (https://uofi.box.com/s/ xjb209go4okmvba69bx405wegrv3rdmf). All the other relevant data are available from the author upon reasonable request.

Received: 13 July 2019; Accepted: 9 July 2020;

Published online: 10 September 2020

\section{References}

1. Dreaden, E. C., Alkilany, A. M., Huang, X., Murphy, C. J. \& El-Sayed, M. A. The golden age: gold nanoparticles for biomedicine. Chem. Soc. Rev. 41, 2740-2779 (2012).

2. Giljohann, D. A. et al. Gold nanoparticles for biology and medicine. Angew. Chem. Int. Ed. 49, 3280-3294 (2010).

3. Halas, N. J., Lal, S., Chang, W.-S., Link, S. \& Nordlander, P. Plasmons in strongly coupled metallic nanostructures. Chem. Rev. 111, 3913-3961 (2011).

4. Jain, P. K., Huang, X., El-Sayed, I. H. \& El-Sayed, M. A. Noble metals on the nanoscale: optical and photothermal properties and some applications in imaging, sensing, biology, and medicine. Acc. Chem. Res. 41, 1578-1586 (2008).

5. Ashley, M. J. et al. Shape and size control of substrate-grown gold nanoparticles for surface-enhanced raman spectroscopy detection of chemical analytes. J. Phys. Chem. C 122, 2307-2314 (2018).

6. Schwartz-Duval, A. S. et al. An anisotropic propagation technique for synthesizing hyperbranched polyvillic gold nanoparticles. Nano Res. 9 , 2889-2903 (2016)

7. Xia, Y. \& Halas, N. J. Shape-controlled synthesis and surface plasmonic properties of metallic nanostructures. MRS Bull. 30, 338-348 (2005).

8. Xiao, J. \& Qi, L. Surfactant-assisted, shape-controlled synthesis of gold nanocrystals. Nanoscale 3, 1383-1396 (2011).

9. Chithrani, B. D., Ghazani, A. A. \& Chan, W. C. Determining the size and shape dependence of gold nanoparticle uptake into mammalian cells. Nano Lett. 6, 662-668 (2006).

10. Chithrani, B. D. \& Chan, W. C. Elucidating the mechanism of cellular uptake and removal of protein-coated gold nanoparticles of different sizes and shapes. Nano Lett. 7, 1542-1550 (2007).

11. Singh, A. V. et al. Review of emerging concepts in nanotoxicology: opportunities and challenges for safer nanomaterial design. Toxicol. Mech, Methods 29, 378-387 (2019).

12. Sonavane, G., Tomoda, K. \& Makino, K. Biodistribution of colloidal gold nanoparticles after intravenous administration: effect of particle size. Colloids Surf. B 66, 274-280 (2008).

13. Hirn, S. et al. Particle size-dependent and surface charge-dependent biodistribution of gold nanoparticles after intravenous administration. Eur. J. Pharm. Biopharm. 77, 407-416 (2011).

14. Zhang, X.-D. et al. In vivo renal clearance, biodistribution, toxicity of gold nanoclusters. Biomaterials 33, 4628-4638 (2012).

15. Liu, J. et al. Passive tumor targeting of renal-clearable luminescent gold nanoparticles: long tumor retention and fast normal tissue clearance. J. Am Chem. Soc. 135, 4978-4981 (2013). 
16. Huang, X., El-Sayed, I. H., Qian, W. \& El-Sayed, M. A. Cancer cell imaging and photothermal therapy in the near-infrared region by using gold nanorods. J. Am. Chem. Soc. 128, 2115-2120 (2006).

17. Gobin, A. M. et al. Near-infrared resonant nanoshells for combined optical imaging and photothermal cancer therapy. Nano Lett. 7, 1929-1934 (2007).

18. Liu, C. L. et al. Insulin-directed synthesis of fluorescent gold nanoclusters: preservation of insulin bioactivity and versatility in cell imaging. Angew. Chem. Int. Ed. 50, 7056-7060 (2011).

19. Purohit, R. \& Singh, S. Fluorescent gold nanoclusters for efficient cancer cell targeting. Int. J. Nanomed. 13, 15 (2018).

20. Cheheltani, R. et al. Tunable, biodegradable gold nanoparticles as contrast agents for computed tomography and photoacoustic imaging. Biomaterials 102, 87-97 (2016).

21. Chhour, P. et al. Effect of gold nanoparticle size and coating on labeling monocytes for CT tracking. Bioconjug. Chem. 28, 260-269 (2016).

22. Pan, D. et al. Molecular photoacoustic imaging of angiogenesis with integrintargeted gold nanobeacons. FASEB J. 25, 875-882 (2011).

23. Pan, D. et al. Near infrared photoacoustic detection of sentinel lymph nodes with gold nanobeacons. Biomaterials 31, 4088-4093 (2010).

24. Yeager, D. et al. in Photons Plus Ultrasound: Imaging and Sensing 2012 82231Q (International Society for Optics and Photonics).

25. Jang, H., Kang, K. \& El-Sayed, M. A. Real-time tracking of the autophagy process in living cells using plasmonically enhanced Raman spectroscopy of fucoidan-coated gold nanoparticles. J. Mater. Chem. B 6, 5460-5465 (2018).

26. DeVetter, B. M. et al. Observation of molecular diffusion in polyelectrolytewrapped SERS nanoprobes. Langmuir 30, 8931-8937 (2014).

27. Wang, X. et al. Evans blue derivative-functionalized gold nanorods for photothermal therapy-enhanced tumor chemotherapy. ACS Appl. Mater. Interfaces 10, 15140-15149 (2018).

28. Ma, Y. et al. Gold nanoshell nanomicelles for potential magnetic resonance imaging, light-triggered drug release, and photothermal therapy. Adv. Funct. Mater. 23, 815-822 (2013)

29. Benton, J. et al. Gold nanoparticles enhance radiation sensitization and suppress colony formation in a feline injection site sarcoma cell line, in vitro. Res. Vet. Sci. 117, 104-110 (2018).

30. Su, X.-Y., Liu, P.-D., Wu, H. \& Gu, N. Enhancement of radiosensitization by metal-based nanoparticles in cancer radiation therapy. Cancer Biol. Med. 11, 86 (2014).

31. Baskar, R., Lee, K. A., Yeo, R. \& Yeoh, K.-W. Cancer and radiation therapy: current advances and future directions. Int. J. Med. Sci. 9, 193 (2012).

32. Misra, S. K. et al. In situ plasmonic generation in functional ionic-goldnanogel scaffold for rapid quantitative bio-sensing. Biosens. Bioelectron. 120, 77-84 (2018).

33. Lee, H.-E. et al. Amino-acid-and peptide-directed synthesis of chiral plasmonic gold nanoparticles. Nature 556, 360 (2018).

34. Yan, F. et al. Controlled synthesis of highly-branched plasmonic gold nanoparticles through peptoid engineering. Nat. Commun. 9, 2327 (2018).

35. Kwon, K. C. et al. Superparamagnetic gold nanoparticles synthesized on protein particle scaffolds for cancer theragnosis. Adv. Mater. 29, 1701146 (2017).

36. Singh, A. V. et al. Seed-mediated synthesis of plasmonic gold nanoribbons using cancer cells for hyperthermia applications. J. Mater. Chem. B 6 , 7573-7581 (2018)

37. Singh, A. V. et al. Anisotropic gold nanostructures: optimization via in silico modeling for hyperthermia. ACS Appl. Nano Mater. 1, 6205-6216 (2018).

38. Wang, J. et al. In vivo self-bio-imaging of tumors through in situ biosynthesized fluorescent gold nanoclusters. Sci. Rep. 3, 1157 (2013)

39. West, A. L. et al. In situ synthesis of fluorescent gold nanoclusters by nontumorigenic microglial cells. ACS Appl. Mater. interfaces 8, 21221-21227 (2016).

40. Zhao, C. et al. Biosynthesized gold nanoclusters and iron complexes as scaffolds for multimodal cancer bioimaging. Small 12, 6255-6265 (2016).

41. Rehman, F. U. et al. Nano in nano: Biosynthesized gold and iron nanoclusters cargo neoplastic exosomes for cancer status biomarking. Nanomed.: Nanotechnol. Biol. Med. 14, 2619-2631 (2018).

42. Singh, A. V. et al. Cancer cells biomineralize ionic gold into nanoparticlesmicroplates via secreting defense proteins with specific gold-binding peptides. Acta Biomater. 71, 61-71 (2018).

43. Drescher, D., Traub, H., Büchner, T., Jakubowski, N. \& Kneipp, J. Properties of in situ generated gold nanoparticles in the cellular context. Nanoscale 9, 11647-11656 (2017).

44. El-Said, W. A., Cho, H. Y., Yea, C. H. \& Choi, J. W. Synthesis of metal nanoparticles inside living human cells based on the intracellular formation process. Adv. Mater. 26, 910-918 (2014).

45. Liu, Z., Hu, C., Li, S., Zhang, W. \& Guo, Z. Rapid intracellular growth of gold nanostructures assisted by functionalized graphene oxide and its application for surface-enhanced Raman spectroscopy. Anal. Chem. 84, 10338-10344 (2012).
46. Shamsaie, A., Jonczyk, M., Sturgis, J. D., Robinson, J. P. \& Irudayaraj, J. Intracellularly grown gold nanoparticles as potential surface-enhanced Raman scattering probes. J. Biomed. Opt. 12, 020502 (2007).

47. Anshup et al. Growth of gold nanoparticles in human cells. Langmuir 21, 11562-11567 (2005).

48. Silvert, P.-Y. \& Tekaia-Elhsissen, K. Synthesis of monodisperse submicronic gold particles by the polyol process. Solid State Ion. 82, 53-60 (1995).

49. Xie, J., Zheng, Y. \& Ying, J. Y. Protein-directed synthesis of highly fluorescent gold nanoclusters. J. Am. Chem. Soc. 131, 888-889 (2009).

50. Singh, A., Patil, R., Kasture, M., Gade, W. \& Prasad, B. Synthesis of Ag-Pt alloy nanoparticles in aqueous bovine serum albumin foam and their cytocompatibility against human gingival fibroblasts. Colloids Surf. B 69, 239-245 (2009).

51. Lin-Vien, D., Colthup, N. B., Fateley, W. G. \& Grasselli, J. G. The Handbook of infrared and Raman characteristic frequencies of organic molecules (Elsevier, 1991).

52. Shehadul Islam, M., Aryasomayajula, A. \& Selvaganapathy, P. R. A review on macroscale and microscale cell lysis methods. Micromachines 8, 83 (2017).

53. Lodish, H. et al. Molecular Cell Biology 4th edn (National Center for Biotechnology Information, Bookshelf, 2000).

54. Dharanivasan, G. et al. DNA templated self-assembly of gold nanoparticle clusters in the colorimetric detection of plant viral DNA using a gold nanoparticle conjugated bifunctional oligonucleotide probe. RSC $A d v$. 6 , 11773-11785 (2016)

55. Shoji, M., Kato, M. \& Hashizume, S. Electrophoretic recovery of proteins from polyacrylamide gel. J. Chromatogr. A 698, 145-162 (1995).

56. Seelert, H. \& Krause, F. Preparative isolation of protein complexes and other bioparticles by elution from polyacrylamide gels. Electrophoresis 29, 2617-2636 (2008).

57. Burgess, R. R. in Methods in Enzymology, vol. 463, 565-572 (Elsevier, 2009)

58. Binder, J. X. et al. COMPARTMENTS: unification and visualization of protein subcellular localization evidence. Database 2014, bau012 https://doi.org/ 10.1093/database/bau012 (2014)

59. Gaudet, P., Livstone, M. S., Lewis, S. E. \& Thomas, P. D. Phylogenetic-based propagation of functional annotations within the Gene Ontology consortium. Brief Bioinforma. 12, 449-462 (2011).

60. Trinick, J. \& Tskhovrebova, L. Titin: a molecular control freak. Trends Cell Biol. 9, 377-380 (1999).

61. Gliem, M., Heupel, W.-M., Spindler, V., Harms, G. S. \& Waschke, J. Actin reorganization contributes to loss of cell adhesion in Pemphigus vulgaris. Am. J. Physiol. 299, C606-C613 (2010).

62. Hunger-Glaser, I., Fan, R. S., Perez-Salazar, E. \& Rozengurt, E. PDGF and FGF induce focal adhesion kinase (FAK) phosphorylation at Ser-910: dissociation from Tyr-397 phosphorylation and requirement for ERK activation. J. Cell. Physiol. 200, 213-222 (2004).

63. Allen, C., Yu, Y., Eisenberg, A. \& Maysinger, D. Cellular internalization of PCL20-b-PEO44 block copolymer micelles. Biochim. Biophys. Acta Biomembr. 1421, 32-38 (1999).

64. Wang, L. H., Rothberg, K. G. \& Anderson, R. G. Mis-assembly of clathrin lattices on endosomes reveals a regulatory switch for coated pit formation. J. Cell Biol. 123, 1107-1117 (1993).

65. Kirchhausen, T., Macia, E. \& Pelish, H. E. Use of dynasore, the small molecule inhibitor of dynamin, in the regulation of endocytosis. Methods Enzymol. 438 77-93 (2008).

66. Sigismund, S. et al. Clathrin-independent endocytosis of ubiquitinated cargos. Proc. Natl Acad. Sci. USA 102, 2760-2765 (2005).

67. Goswami, N. et al. Luminescent metal nanoclusters with aggregation-induced emission. J. Phys. Chem. Lett. 7, 962-975 (2016)

68. Huang, X., Jain, P. K., El-Sayed, I. H. \& El-Sayed, M. A. Plasmonic photothermal therapy (PPTT) using gold nanoparticles. Lasers Med. Sci. 23, 217 (2008).

69. Qin, Z. et al. Quantitative comparison of photothermal heat generation between gold nanospheres and nanorods. Sci. Rep. 6, 1-13 (2016)

70. Wu, Y. et al. Gold nanorod photothermal therapy alters cell junctions and actin network in inhibiting cancer cell collective migration. ACS Nano $\mathbf{1 2}$ 9279-9290 (2018).

71. Coakley, W. Hyperthermia effects on the cytoskeleton and on cell morphology. Symposia of the Society for Experimental Biology, 41, 187-211 (1986).

72. Amendola, V. \& Meneghetti, M. Laser ablation synthesis in solution and size manipulation of noble metal nanoparticles. Phys. Chem. Chem. Phys. 11, 3805-3821 (2009)

73. Scaramuzza, S., Zerbetto, M. \& Amendola, V. Synthesis of gold nanoparticles in liquid environment by laser ablation with geometrically confined configurations: insights to improve size control and productivity. J. Phys. Chem. C 120, 9453-9463 (2016)

74. Zhang, J., Chaker, M. \& Ma, D. Pulsed laser ablation based synthesis of colloidal metal nanoparticles for catalytic applications. J. Colloid Interface Sci. 489, 138-149 (2017). 


\section{Acknowledgements}

We thank Frederick Seitz Materials Research Laboratory Central Research Facilities, and the Beckman research institute. Authors gratefully acknowledge funding from the University of Illinois at Urbana-Champaign, National Institute of Health (R03EB028026), and National Science Foundation. A.S.S.-D. gratefully acknowledges fellowship funding from the American Heart Association (16PRE30150004) and the NIH (5T32CA19656105). Authors also acknowledge the receipt of funding from University of Maryland Baltimore and University of Maryland Baltimore County.

\section{Author contributions}

D.P. and A.S.S.-D. conceived the main idea and designed the work. A.S.S.-D. performed all experimental work. C.J.K. lead animal experiments. E.A.D. performed and assisted with Raman cell imaging. I.S. conducted flow cytometry experiments and conducted $\mathrm{UV}-\mathrm{V}$ is experiments to address reviewer comments. E.V.J. conducted hyperspectral darkfield imaging. T.L.K. assisted with cell culture and performed MTT assay. S.F. and A.H. assisted C.J.K. and A.S.S.-D. with animal experiments and data processing. P.M. majorly contributed to followup experiments and substantial revisions necessary to address reviewer comments for resubmission. L.W.D. oversaw the animal experiments and contribuited towards the manuscript preparation. D.P. is the academic supervisor of the work and substantially revised the paper. All the authors analyzed, interpreted the results, contributed to drafting the paper, and provided their consent to the last draft of the manuscript.

\section{Competing interests}

D.P. is the founder/co-founder of three University-based start-ups. None of these entities, however, supported this work. All the authors thus declare no competing interests.

\section{Additional information}

Supplementary information is available for this paper at https://doi.org/10.1038/s41467020-17595-6.

Correspondence and requests for materials should be addressed to D.P.

Peer review information Nature Communications thanks Ajay Singh, Zhiming Liu and the other anonymous reviewer(s) for their contribution to the peer review of this work.

Reprints and permission information is available at http://www.nature.com/reprints

Publisher's note Springer Nature remains neutral with regard to jurisdictional claims in published maps and institutional affiliations.

(c) (i) Open Access This article is licensed under a Creative Commons Attribution 4.0 International License, which permits use, sharing, adaptation, distribution and reproduction in any medium or format, as long as you give appropriate credit to the original author(s) and the source, provide a link to the Creative Commons license, and indicate if changes were made. The images or other third party material in this article are included in the article's Creative Commons license, unless indicated otherwise in a credit line to the material. If material is not included in the article's Creative Commons license and your intended use is not permitted by statutory regulation or exceeds the permitted use, you will need to obtain permission directly from the copyright holder. To view a copy of this license, visit http://creativecommons.org/ licenses/by/4.0/

(c) The Author(s) 2020 